\title{
A new protocol for the synthesis of primary, secondary and tertiary anthranilamides utilizing $N$-(2-aminoarylacyl)benzotriazoles
}

\author{
Nevin Kanışkan, Şule Kökten, and İlhami Çelik* \\ Department of Chemistry, Faculty of Science, Anadolu University, Eskişehir-26470, Turkey \\ E-mail: ilcelik@anadolu.edu.tr
}

\begin{abstract}
A convenient route for efficient conversion of unprotected anthranilic acids into the corresponding $N$-(2-aminoarylacyl)benzotrazoles is described. $N$-(2-Aminoarylacyl)benzotrazoles have been successfully used to synthesize primary, secondary and tertiary anthranilamides in high yields (71-96\%).
\end{abstract}

Keywords: Anthranilic acid, benzotriazole, $N$-(2-aminoarylacyl)benzotrazoles, anthranilamides

\section{Introduction}

Amides are of great importance in synthetic organic chemistry because of the numerous natural products (e.g. peptides and proteins) and potential drug compounds that contain amide bond, like acetaminophen, an analgesic; lidocaine (Xylocaine), a local anaesthetic and loperamide (Imodium AD), an anti-diarrheal. Amides are also used in different areas of industry ${ }^{1}$ (e.g. plastic and rubber industry, and paper industry). As a consequence, much effort has been put onto synthesize new derivatives of amides using mild ways. Anthranilamides are an example of useful compounds containing amide bond since they are used as a starting compound or an intermediate in the synthesis of biologically important compounds such as quinazolones, ${ }^{2}$ quinazolinones ${ }^{3 a-c}$ and benzoxazinones. ${ }^{3 c, 4}$ In addition, some amide derivatives of anthranilic acid show biological activities $^{5}$ or they are the starting materials for o-aminonitriles which are versatile synthons to get some useful heterocycles. ${ }^{6}$ Literature methods for synthesizing anthranilamides are: (i) the reaction of anthranilic acid with amine in the presence of various reagents $\left(\mathrm{SOCl}_{2},{ }^{7 \mathrm{a}} \mathrm{EDC} /\right.$ $\mathrm{HOBt},{ }^{7 \mathrm{~b}} \mathrm{CDI},{ }^{7 \mathrm{c}} \mathrm{BAC},{ }^{7 \mathrm{~d}} \mathrm{HOSu} / \mathrm{DCC},{ }^{7 \mathrm{e}} \mathrm{DCC} / \mathrm{HOBt}^{7 \mathrm{f}}$ ) (ii) the reaction of anthranilesters with amines, ${ }^{8}$ (iii) the catalytically reduction of 2-nitrobenznitriles, ${ }^{9}$ (iv) the reaction of 2-nitrobenzoic acid activated by $\mathrm{SOCl}_{2}$ with an amine, and then catalytically reduction of nitro group, ${ }^{7 f, 10}$ and (v) the reaction of isatoic anhydride with various amines ${ }^{7 c, f, 11,12}$ (Scheme 1). These methods developed to get anthranilamides have some disadvantages like being lack of wide generality in use, having harsh reaction conditions or complex reaction steps. Conventional activation of a 
carboxyl group using thionyl chloride cannot be safely employed in the presence of a free amino group. $^{7 \mathrm{a}}$ The reactions having coupling reagents require anhydrous reaction conditions and activated anthranilic acid intermediates cannot usually be stored, handled in moist air or isolated. ${ }^{7 b, e, f}$ In addition, the method including CDI coupling with amine needs using of phosgene in the preparation of CDI. ${ }^{7 \mathrm{c}}$ The reaction of anthranilesters with amines, on the other hand, requires harsh reaction conditions. ${ }^{8}$ Moreover, the synthesis of anthranilamides from 2nitrobenzonitriles involves the reduction of nitro group and the hydrolysis of nitrile group. The difficulty in purification of the products cause low yields. ${ }^{9}$ The synthesis of anthranilamides from 2-nitro benzoic acids also has some disadvantages like the difficulty in activation of carboxylic acid group with thionyl chloride and purification of products obtained by the reduction of nitro group. ${ }^{10}$ The reaction of isatoic anhydride with amine is a well-known general method for the preparation of anthranilamides. However, this method requires handling of phosgene in the preparation of isatoic anhydride. ${ }^{11}$ Therefore, newer and versatile methods having simple reaction procedure and reagents to prepare these compounds would be advantageous.

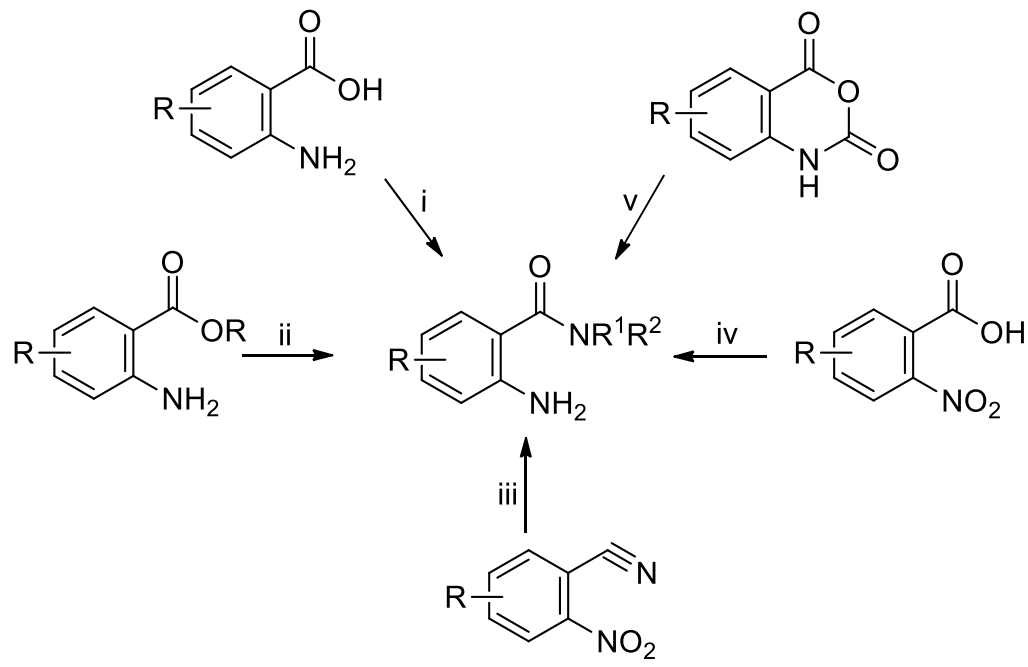

Scheme 1. Literature methods for synthesizing anthranilamides.

Being one of the versatile benzotriazole intermediates, $N$-acylbenzotriazoles have found wide application in the acylation of various nucleophiles because of the good leaving ability of benzotriazole group. They are widely employed in heterocyclic synthesis, ${ }^{13 a, b} \mathrm{~N}$-acylation, ${ }^{14 a-c} \mathrm{C}$ acylation, ${ }^{14 b, 15 a, b}$ S-acylation ${ }^{14 b, 16 a, b}$ and O-acylation. ${ }^{17}$ Unlike the conventional acylating agents which are unstable to moisture and difficult to prepare and store at room temperature, $\mathrm{N}$ acylbenzotriazoles are stable crystalline compounds and they are easy to use and store at room temperature. ${ }^{14 a}, 18$ They can be prepared directly from carboxylic acids even in cases where an acid sensitive functionality is present. In literature, $\mathrm{N}$-acylbenzotriazoles are synthesized from the reaction of carboxylic acids (i) with 1-(methylsulfonyl)benzotriazole in the presence of triethylamine $^{14 \mathrm{a}}$ or (ii) with the excess amount of benzotriazole in the presence of 
thionylchloride ${ }^{19}$ and from the reaction of various aldehydes with $N$-chlorobenzotriazole. ${ }^{20}$ So far today, various $N$-acylbenzotriazole derivatives have been synthesized from alkyl-, aryl-, heterocyclic- and unsaturated carboxylic acids or carboxylic acids with different functionality. However, there have been no reported studies on the direct synthesis of $\mathrm{N}$-acylbenzotriazoles using anthranilic acids. Anthranilic acid component has two different functional groups $(\mathrm{COOH}$ and $\mathrm{NH}_{2}$ ). The presence of a free amino group makes the activation of carboxylic group difficult and decreases the yield. Therefore, the protection and subsequent deprotection of amino group are necessary to regenerate the amine functionality. However, the use of protecting groups has some drawbacks, like being not economical and causing time loss.

We now report a mild one pot procedure for efficient conversion of anthranilic acids into the corresponding $\mathrm{N}$-acylbenzotriazoles having free amino group and subsequent reaction of $\mathrm{N}$ acylbenzotriazoles with ammonia, primary and secondary amines to afford substantial anthranilamides.

\section{Results and Discussion}

As mentioned before, $N$-acylbenzotriazoles are highly efficient acylating agents to prepare various kinds of compounds comparing to the conventional acylating agents which are mostly sensitive to air and so, difficult to prepare and store at room temperature. Moreover, as an auxiliary group benzotriazole can be recovered almost quantitatively after the reaction. Therefore, this method has also the potential of recycling the starting material.

In the present work, anthranilic acid derivatives were successfully converted into the corresponding $\mathrm{N}$-acylbenzotriazoles in the presence of free amino group (Scheme 2). Activation of anthranilic acid with benzotriazole was succeeded by using mild DCC coupling condition. The presence of ortho-substituted free amine functionality in the molecules did not cause any troubles except for $\mathbf{2 e}$ and $\mathbf{2 f}$. The resulting compounds $\mathbf{2 a - 0}$ were successfully isolated and purified by column chromatography as stable, yellow colored solids in $14-93 \%$ yields (Table 1). All of the $\mathrm{N}$-acylbenzotriazole derivatives prepared are novel compounds and structures of products 2a-o were fully characterized by IR, ${ }^{1} \mathrm{H}$ NMR and ${ }^{13} \mathrm{C}$ NMR spectroscopy. Elemental analysis and mass spectroscopy also supported the proposed structures. The most characteristic ${ }^{1} \mathrm{H}-\mathrm{NMR}$ data indicating that benzotriazole attached to the molecules are the signals which were observed for the protons on the benzotriazole ring as doublets at $8.37-8.17 \mathrm{ppm}$ and as triplets at $7.69-7.55$

ppm. In addition to the ${ }^{1} \mathrm{H}-\mathrm{NMR}$ spectral data, 12 signals were observed for the carbons in aromatic rings of the products in the ${ }^{13} \mathrm{C}-\mathrm{NMR}$ spectra. 


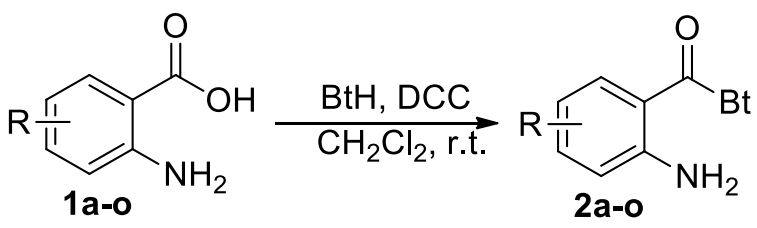

Scheme 2. Synthesis of $N$-(2-aminoarylacyl)benzotriazoles (2a-o).

Table 1. Synthesized $N$-(2-aminoarylacyl)benzotriazoles 2a-o from the proposed method

\begin{tabular}{cccccccc}
\hline & $\mathrm{R}$ & $\begin{array}{c}\text { Yield } \\
(\%)\end{array}$ & $\begin{array}{c}\mathrm{Mp} \\
\left({ }^{\circ} \mathrm{C}\right)\end{array}$ & $\mathrm{R}$ & $\begin{array}{c}\text { Yield } \\
(\%)\end{array}$ & $\begin{array}{c}\mathrm{Mp} \\
\left({ }^{\circ} \mathrm{C}\right)\end{array}$ \\
\hline $\mathbf{a}$ & $\mathrm{H}$ & 78 & $132-133$ & $\mathbf{i}$ & 5-bromo & 53 & $174-176$ \\
$\mathbf{b}$ & 3-methyl & 82 & $99-100$ & $\mathbf{j}$ & 5-iodo & 55 & $138-140$ \\
$\mathbf{c}$ & 4-methyl & 76 & $135-136$ & $\mathbf{k}$ & 3,5-dichloro & 93 & $170-172$ \\
$\mathbf{d}$ & 5-methyl & 40 & $104-106$ & $\mathbf{1}$ & 3,5-dibromo & 83 & $174-176$ \\
$\mathbf{e}$ & 6-methyl & 25 & $106-108$ & $\mathbf{m}$ & 3,5-diiodo & 64 & decomposed $>$ \\
$\mathbf{f}$ & 5-methoxy & 14 & $107-109$ & $\mathbf{n}$ & 4,5-dimethoxy & 70 & $93-95$ \\
$\mathbf{g}$ & 4-chloro & 92 & $160-162$ & $\mathbf{0}$ & $\begin{array}{c}\text { 3,4,5- } \\
\text { trimethoxy }\end{array}$ & 75 & $115-117$ \\
$\mathbf{h}$ & 5-chloro & 74 & $153-155$ & & & & \\
\hline
\end{tabular}

During the preparation of $N$-(2-aminoarylacyl)benzotriazoles 2a-o, it was noticed that a byproduct 2' (Figure 1) was formed with the desired products because of the nature and position of the substituents in the corresponding anthranilic acids. The yields of by-products were almost negligible except for $\mathbf{2 e}$ and $\mathbf{2 f}$. The by-products $2 \mathbf{e}^{\prime}$ and $\mathbf{2 \mathbf { f } ^ { \prime }}$ were isolated by column chromatography in $27 \%$ and 42 yields respectively. Their structures were supported by ${ }^{1} \mathrm{H}$ and ${ }^{13} \mathrm{C}$ NMR spectra. The expected signals which belong to benzotriazole ring weren't observed in ${ }^{1} \mathrm{H}$ and ${ }^{13} \mathrm{C}$ NMR spectra. Moreover, for the two carbonyl carbons in the structures, two signals were observed in ${ }^{13} \mathrm{C}$ NMR spectra. MS spectra also support the structures of these side products. Steric hindrance of the methyl group, which is in ortho position to the carboxylic acid, caused the low yield of the desired product $2 \mathbf{e}$. In case of $\mathbf{2 f}$, the methoxy group present in para position to amino group increased nucleofilicity of amino group and caused the formation of by-product. 


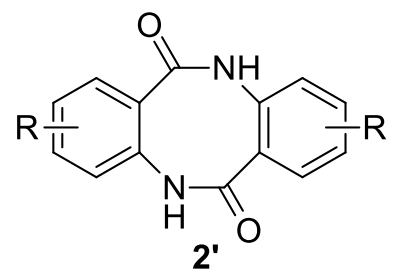

Figure 1. The by-product produced during the activation of anthranilic acids with benzotriazole.

The reaction of $\mathrm{N}$-(2-aminoarylacyl)benzotriazoles with ammonia, various primary and secondary amines gives primary, secondary and tertiary anthranilamides. In spite of having free amino groups, no complications were observed and the reactions were rather straight forward. Therefore, anthranilamides were synthesized in high yields (71-96\%). Spectroscopic studies have provided strong evidence that amides formed. Especially, the disappearance of the benzotriazole signals in the aromatic region of ${ }^{1} \mathrm{H}-\mathrm{NMR}$ spectra indicates the loss of the benzotriazolyl group during the reaction and shows the formation of amides. Characteristic amide peaks were observed for all compounds on IR spectra. Moreover, mass spectra show that the expected molecular ion and the fragmentation are in accordance with the proposed structures.

Primary anthranilamides were efficiently synthesized by treatment of $\mathrm{N}$-acylbenzotriazoles with excess ammonium hydroxide (27\% aqueous solution) in THF at $0{ }^{\circ} \mathrm{C}$ for 2 hours (Scheme 3 ). The products were isolated by column chromatography in $82-96 \%$ yields (Table 2) and characterized spectrally and by elemental analyses. The ${ }^{1} \mathrm{H}-\mathrm{NMR}$ spectrum showed two broad signals for each proton of amino groups attached to the carbonyl group at 8.06-7.58 ppm and 7.47-6.44 ppm, which are in agreement with the reported values for these types of compounds. ${ }^{21 \mathrm{a}, \mathrm{b}}$ Similarly, the signals in the ${ }^{13} \mathrm{C}-\mathrm{NMR}$ spectra for carbonyl carbons were observed at 172.8-170.5 ppm, apart from six carbon signals for aromatic ring in the aromatic region. In addition to spectral and elemental analysis, the melting points of the products were measured (Table 2).

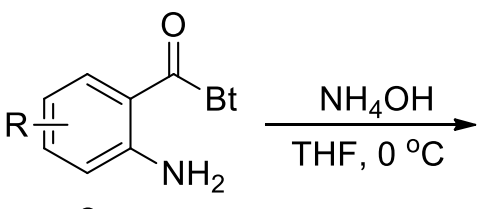

2<smiles>NC(=O)c1cc[R]#cc1N</smiles>

3a-g

Scheme 3. Synthesis of primary amides (3a-g) from $N$-(2-aminoarylacyl)benzotriazoles. 
Table 2. Preparation of primary amides 3a-g

\begin{tabular}{ccccc}
\hline & $\mathrm{R}$ & $\begin{array}{c}\text { Yield } \\
(\%)\end{array}$ & $\begin{array}{c}\text { Lit. Yield } \\
(\%)\end{array}$ & $\begin{array}{c}\mathrm{mp} \\
\left({ }^{\circ} \mathrm{C}\right)\end{array}$ \\
\hline $\mathbf{a}$ & $\mathrm{H}$ & 96 & $90^{\text {(Ref. 22) }}$ & $112-113$ \\
$\mathbf{b}$ & 4-methyl & 93 & $86^{\text {(Ref. 21b) }}$ & $148-149$ \\
$\mathbf{c}$ & 4-chloro & 88 & $83^{\text {(Ref. 23) }}$ & $180-182$ \\
$\mathbf{d}$ & 5-bromo & 93 & $100^{\text {(Ref. 24) }}$ & $185-187$ \\
$\mathbf{e}$ & 5-iodo & 89 & $82^{\text {(Ref. 25) }}$ & $177-178$ \\
$\mathbf{f}$ & 4,5-dimethoxy & 82 & $100^{\text {(Ref. 26) }}$ & $148-149$ \\
g & 3,5-dibromo & 85 & $54^{\text {(Ref. 27) }}$ & $214-215$ \\
\hline
\end{tabular}

Various primary amines were treated with anthranilbenzotriazoles in dichloromethane to give a series of secondary anthranilamides 4a-i (Scheme 4). The reactions were completed in 2-6 hours at room temperature with high yields (71-95\%). The synthesized compounds gave satisfactory proton and carbon NMR data. The most characteristic signal for the secondary amides is the broad singlet which was observed at $6.35-5.82 \mathrm{ppm}$ for the protons of the amide nitrogen atom in ${ }^{1} \mathrm{H}-\mathrm{NMR}$. Elemental analysis, IR and mass spectroscopy also supported the structures of $\mathbf{4 a - i}$. The isolated yields, literature yields and observed melting points were summarized in Table 3.

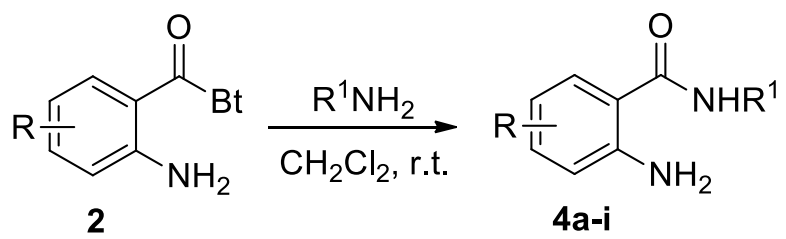

Scheme 4. Synthesis of secondary amides (4a-i) from $N$-(2-aminoarylacyl)benzotriazoles.

Table 3. Preparation of secondary amides $\mathbf{4 a - i}$

\begin{tabular}{cccccc}
\hline & $\mathrm{R}$ & $\mathrm{R} 1$ & $\begin{array}{c}\text { Yield } \\
(\%)\end{array}$ & $\begin{array}{c}\text { Lit. Yield } \\
(\%)\end{array}$ & $\begin{array}{c}\mathrm{mp} \\
\left({ }^{\circ} \mathrm{C}\right)\end{array}$ \\
\hline $\mathrm{a}$ & $\mathrm{H}$ & benzyl & 71 & $95^{\text {(Ref. 28) }}$ & $121-122$ \\
$\mathrm{~b}$ & 3-methyl & n-propyl & 79 & $65^{\text {(Ref. 29) }}$ & $105-106$ \\
$\mathrm{c}$ & 4- methyl & cyclohexyl & 86 & novel & $150-151$ \\
$\mathrm{~d}$ & 5- methyl & cyclohexyl & 86 & novel & $201-202$ \\
$\mathrm{e}$ & 5-methoxy & n-propyl & 82 & novel & $72-74$ \\
$\mathrm{f}$ & 4-chloro & methyl & 93 & $74^{\text {(Ref. 30) }}$ & $96-97$ \\
$\mathrm{~g}$ & 5-bromo & isopropyl & 87 & novel & $188-189$ \\
h & 3,5-dichloro & n-propyl & 95 & novel & $143-144$ \\
$\mathrm{i}$ & 4,5-dimethoxy & methyl & 84 & $80^{\text {(Ref. 31) }}$ & $117-119$ \\
\hline
\end{tabular}


Treatment of $\mathrm{N}$-(2-aminoarylacyl)benzotriazoles with secondary amines in $\mathrm{CH}_{2} \mathrm{Cl}_{2}$ at room temperature gave the corresponding tertiary amides in excellent yields (77-96\%) (Scheme 5, Table 4). Structures of the products were confirmed by the results of NMR measurements. The most important evidence proving the formation of tertiary amides is the signals which were observed for the substituents attached to the amide nitrogen atom. Moreover, no broad singlet was observed except for the one accounting for the amino group on the anthranilic acid ring.

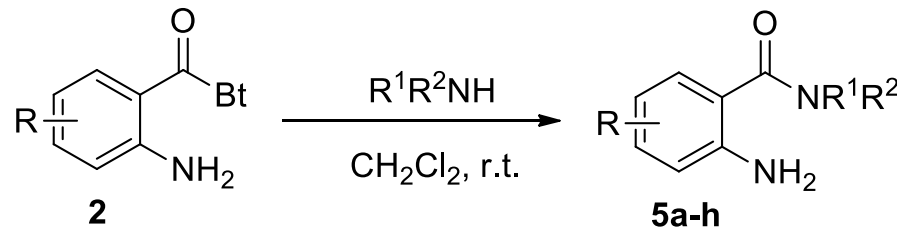

Scheme 5. Synthesis of tertiary amides (5a-h) from $N$-(2-aminoarylacyl)benzotriazoles.

Table 4. Preparation of Tertiary Amides 5a-h

\begin{tabular}{lccccc}
\hline & $\mathrm{R}$ & $\mathrm{R}^{1}+\mathrm{R}^{2}$ & $\begin{array}{c}\text { Yield } \\
(\%)\end{array}$ & $\begin{array}{c}\text { Lit. yield } \\
(\%)\end{array}$ & $\begin{array}{c}\mathrm{mp} \\
\left({ }^{\circ} \mathrm{C}\right)\end{array}$ \\
\hline a & H & $\left(-\mathrm{CH}_{2}\right)_{5}$ & 90 & $83^{\text {(Ref. 31) }}$ & $74-76$ \\
b & 3-methyl & $\left(-\mathrm{CH}_{2}\right)_{5}$ & 86 & $98^{\text {(Ref. 31) }}$ & $80-81$ \\
c & 6- methyl & $\left(-\mathrm{CH}_{2}\right)_{4}$ & 94 & novel & $78-79$ \\
d & 5-chloro & $\left(-\mathrm{CH}_{2}\right)_{4} \mathrm{O}$ & 90 & novel & $130-131$ \\
e & 5-iodo & $\left(-\mathrm{CH}_{2}\right)_{4}$ & 89 & novel & $134-135$ \\
$\mathbf{f}$ & 3,5-dibromo & $\left(-\mathrm{CH}_{2} \mathrm{CH}_{3}\right)_{2}$ & 89 & novel & oil \\
g & 3,5-diiodo & $\left(-\mathrm{CH}_{2}\right)_{4} \mathrm{O}$ & 96 & novel & $128-130$ \\
h & 3,4,5-trimethoxy & $\left(-\mathrm{CH}_{2} \mathrm{CH}_{3}\right)_{2}$ & 93 & novel & oil \\
\hline
\end{tabular}

\section{Conclusions}

In conclusion, a series of $N$-(2-aminoarylacyl)benzotriazoles were synthesized from unprotected orthoaminoarylcarboxylic acids. By the treatment of synthesized $\mathrm{N}$-(2aminoarylacyl)benzotriazoles with ammonia, primary and secondary amines, we have developed a new and practical protocol for the synthesis of anthranilamides. Advantages of this method compared to the existing methodologies are: providing neutral reaction conditions for the compounds possessing acid - base sensitive substituents, using $N$-acylbenzotriazoles which are more useful and stable than the corresponding acyl halides or other activated derivatives of carboxylic acids, general application and good yields that are comparable to the literature. 


\section{Experimental Section}

General. Column chromatography was conducted on silica gel $70-230$ meshes. Melting points were determined on a Mettler Toledo apparatus and uncorrected. All NMR spectra were recorded on a Bruker Advance 500 DPX spectrometer in $\mathrm{CDCl}_{3}$ or DMSO- $d_{6}$ with TMS as the internal reference for ${ }^{1} \mathrm{H}(500 \mathrm{MHz})$ and ${ }^{13} \mathrm{C}(125 \mathrm{MHz})$. Reagents obtained commercially were used without further purification. MS analyses were obtained with a GC-MS/MS (Thermo Finnigan PolarisQ). Elemental analysis was performed for all compounds with a Thermo Finnigan Flash EA 1112 instrument. FTIR spectra were determined on a PerkinElmer 100 FTIR spectrometer.

General experimental procedure for the preparation of $\mathrm{N}$-(2-aminoarylacyl)benzotriazoles (2a-p). A mixture of appropriate anthranilic acid $(6 \mathrm{mmol})$, benzotriazole $(5 \mathrm{mmol}, 0.595 \mathrm{gr})$ and DCC (7 mmol, 1.44 gr) was stirred in $\mathrm{CH}_{2} \mathrm{Cl}_{2}(10 \mathrm{~mL})$ overnight at room temperature. After completion of the reaction, the solvent was removed under reduced pressure. Purification of the residue by column chromatography over silica gel with EtOAc/ n-Hexane (1:3) or $\mathrm{CH}_{2} \mathrm{Cl}_{2}$ gave the desired product.

(2-Aminophenyl) (benzotriazole-1-yl)methanone (2a). Yellow solid (0.928 g, 78\%); mp 132$133{ }^{\circ} \mathrm{C}$; IR $v_{\max }(\mathrm{KBr}) 3476,3356,1680 \mathrm{~cm}^{-1} ;{ }^{1} \mathrm{H} \mathrm{NMR}\left(\mathrm{CDCl}_{3}\right) \delta 8.29(\mathrm{~d}, J 8.3 \mathrm{~Hz}, 1 \mathrm{H}), 8.19$ $(\mathrm{d}, J 8.3 \mathrm{~Hz}, 1 \mathrm{H}), 8.07(\mathrm{~d}, J 8.2 \mathrm{~Hz}, 1 \mathrm{H}), 7.70(\mathrm{t}, J 7.7 \mathrm{~Hz}, 1 \mathrm{H}), 7.55(\mathrm{t}, J 7.7 \mathrm{~Hz}, 1 \mathrm{H}), 7.43(\mathrm{t}, J$ $7.7 \mathrm{~Hz}, 1 \mathrm{H}), 6.84-6.79(\mathrm{~m}, 2 \mathrm{H}), 5.81(\mathrm{br} \mathrm{s}, 2 \mathrm{H}) ;{ }^{13} \mathrm{C}$ NMR $\delta 167.3,151.9,145.7,135.4,134.3$, 132.7, 129.9, 125.9, 120.2, 117.0, 116.5, 114.5, 111.8; MS (EI): $\mathrm{m} / z$ [ $\left.\mathrm{M}^{+}\right]$calcd. for $\mathrm{C}_{13} \mathrm{H}_{10} \mathrm{~N}_{4} \mathrm{O}$ : 238.08, found: 238.0; Anal. Calc. for $\mathrm{C}_{13} \mathrm{H}_{10} \mathrm{~N}_{4} \mathrm{O}: \mathrm{C}, 65.54 ; \mathrm{H}, 4.23$; N, 23.52. Found: C, 65.63; $\mathrm{H}, 4.28 ; \mathrm{N}, 23.63$.

(2-Amino-3-methylphenyl) (benzotriazole-1-yl)methanone (2b). Yellow solid (1.03 g, 82\%); mp 99-100 ${ }^{\circ} \mathrm{C}$; IR $v_{\max }(\mathrm{KBr}) 3473,3369,1670 \mathrm{~cm}^{-1} ;{ }^{1} \mathrm{H} \mathrm{NMR}\left(\mathrm{CDCl}_{3}\right) \delta 8.26(\mathrm{~d}, J 8.3 \mathrm{~Hz}, 1 \mathrm{H})$, $8.18(\mathrm{~d}, J 8.3 \mathrm{~Hz}, 1 \mathrm{H}), 7.89(\mathrm{~d}, J 8.2 \mathrm{~Hz}, 1 \mathrm{H}), 7.68(\mathrm{t}, J 7.7 \mathrm{~Hz}, 1 \mathrm{H}), 7.54(\mathrm{t}, J 7.7 \mathrm{~Hz}, 1 \mathrm{H}), 7.34$ $(\mathrm{d}, J 7.2 \mathrm{~Hz}, 1 \mathrm{H}), 6.74(\mathrm{t}, J 7.7 \mathrm{~Hz}, 1 \mathrm{H}), 5.34(\mathrm{br} \mathrm{s}, 2 \mathrm{H}), 2.25(\mathrm{~s}, 3 \mathrm{H}) ;{ }^{13} \mathrm{C}$ NMR $\delta 167.8,150.3$, 145.7, 136.1, 132.7, 132.4, 129.8, 125.9, 123.2, 120.1, 115.8, 114.4, 111.3, 17.5; MS (EI): $\mathrm{m} / \mathrm{z}$ [M $\mathrm{M}^{+}$calcd. for $\mathrm{C}_{14} \mathrm{H}_{12} \mathrm{~N}_{4} \mathrm{O}: 252.10$, found: 252.0; Anal. Calc. for $\mathrm{C}_{14} \mathrm{H}_{12} \mathrm{~N}_{4} \mathrm{O}: \mathrm{C}, 66.65 ; \mathrm{H}, 4.79$; N, 22.21. Found: C, 66.72; H, 4.92; N, 22.38.

(2-Amino-4-methylphenyl) (benzotriazole-1-yl)methanone (2c). Yellow solid (0.958 g, 76\%); mp 135-136 ${ }^{\circ} \mathrm{C}$; IR $v_{\max }(\mathrm{KBr}) 3465,3351,1675 \mathrm{~cm}^{-1} ;{ }^{1} \mathrm{H} \mathrm{NMR}\left(\mathrm{CDCl}_{3}\right) \delta 8.24(\mathrm{~d}, J 8.3 \mathrm{~Hz}$, $1 \mathrm{H}), 8.17(\mathrm{~d}, J 8.3 \mathrm{~Hz}, 1 \mathrm{H}), 7.96(\mathrm{~d}, J 8.3 \mathrm{~Hz}, 1 \mathrm{H}), 7.66(\mathrm{t}, J 7.7 \mathrm{~Hz}, 1 \mathrm{H}), 7.52(\mathrm{t}, J 7.6 \mathrm{~Hz}, 1 \mathrm{H})$, 6.61-6.59 (m, 2H), 6.22 (br s, 2H), $2.36(\mathrm{~s}, 3 \mathrm{H}) ;{ }^{13} \mathrm{C}$ NMR $\delta$ 168.0, 153.4, 147.7, 146.5, 135.0, 133.6, 130.5, 126.5, 120.7 118.8, 117.7, 115.1, 109.9, 22.0; MS (EI): $\mathrm{m} / \mathrm{z}\left[\mathrm{M}^{+}\right]$calcd. for $\mathrm{C}_{14} \mathrm{H}_{12} \mathrm{~N}_{4} \mathrm{O}$ : 252.10, found: 252.0; Anal. Calc. for $\mathrm{C}_{14} \mathrm{H}_{12} \mathrm{~N}_{4} \mathrm{O}$ : C, 66.65; H, 4.79; N, 22.21 . Found: C, 66.57; H, 4.76; N, 22.22 .

(2-Amino-5-methylphenyl) (1H-benzotriazole-1-yl)methanone (2d). Yellow solid (0,504 g, 40\%); mp 104-106 ${ }^{\circ} \mathrm{C}$; IR $v_{\max }(\mathrm{KBr}) 3477,3367,1682 \mathrm{~cm}^{-1} ;{ }^{1} \mathrm{H}$ NMR $\left(\mathrm{CDCl}_{3}\right) \delta 8.26(\mathrm{~d}, J 8.3$ $\mathrm{Hz}, 1 \mathrm{H}), 8.18(\mathrm{~d}, J 8.3 \mathrm{~Hz}, 1 \mathrm{H}), 7.82($ br s, 1H), 7.69 (t, $J 7.7 \mathrm{~Hz}, 1 \mathrm{H}), 7.54(\mathrm{t}, J 7.7 \mathrm{~Hz}, 1 \mathrm{H})$, 
$7.25(\mathrm{~d}, J 8.4 \mathrm{~Hz}, 1 \mathrm{H}), 6.75(\mathrm{~d}, J 8.4 \mathrm{~Hz}, 1 \mathrm{H}), 5.67$ (br s, 2H), 2.29 (s, 3H); ${ }^{13} \mathrm{C}$ NMR $\delta 168.3$, $150.8,146.6,137.5,134.4,133.5,130.6,126.6,120.8,117.8,115.2,112.5,20.5 ; \mathrm{MS}(\mathrm{EI}): \mathrm{m} / \mathrm{z}$ $\left[\mathrm{M}^{+}\right]$calcd. for $\mathrm{C}_{14} \mathrm{H}_{12} \mathrm{~N}_{4} \mathrm{O}$ : 252.10, found: 252.0; Anal. Calc. for $\mathrm{C}_{14} \mathrm{H}_{12} \mathrm{~N}_{4} \mathrm{O}: \mathrm{C}, 66.65 ; \mathrm{H}, 4.79$; N, 22.21. Found: 66.91; H, 4.90; N, 22.25.

(2-Amino-6-methylphenyl) (benzotriazole-1-yl)methanone (2e). Yellow solid (0.315 g, 25\%); mp 106-108 ${ }^{\circ} \mathrm{C}$; IR v $v_{\max }(\mathrm{KBr}) 3436,3369,1707 \mathrm{~cm}^{-1} ;{ }^{1} \mathrm{H} \mathrm{NMR}\left(\mathrm{CDCl}_{3}\right) \delta 8.37(\mathrm{~d}, J 8.5 \mathrm{~Hz}$, $1 \mathrm{H}), 8.18(\mathrm{~d}, J 8.0 \mathrm{~Hz}, 1 \mathrm{H}), 7.73(\mathrm{t}, J 8.0 \mathrm{~Hz}, 1 \mathrm{H}), 7.58(\mathrm{t}, J 8.3 \mathrm{~Hz}, 1 \mathrm{H}), 7.26(\mathrm{t}, J 7.8 \mathrm{~Hz}, 1 \mathrm{H})$, $6.73(\mathrm{~d}, J 7.5 \mathrm{~Hz}, 1 \mathrm{H}), 6.70(\mathrm{~d}, J 8.0 \mathrm{~Hz}, 1 \mathrm{H}), 4.14$ (br s, 2H), 2.11 (s, 3H); ${ }^{13} \mathrm{C}$ NMR $\delta 168.9$, $146.4,145.6,137.5,132.2$, 131.3, 130.5, 126.5, 120.6, 120.4, 119.3, 114.5, 114.4, 20.4; MS (EI): $m / z$ [ $\left.\mathrm{M}^{+}\right]$calcd. for $\mathrm{C}_{14} \mathrm{H}_{12} \mathrm{~N}_{4} \mathrm{O}$ : 252.10, found: 252.0; Anal. Calc. for $\mathrm{C}_{14} \mathrm{H}_{12} \mathrm{~N}_{4} \mathrm{O}: \mathrm{C}, 66.65 ; \mathrm{H}$, 4.79; N, 22.21. Found: C, 66.50; H, 4.80; N, 22.43.

(2-Amino-5-methoxyphenyl) (benzotriazole-1-yl)methanone (2f). Yellow solid (0.188 g, 14\%); mp 107-109 ${ }^{\circ} \mathrm{C}$; IR v $v_{\max }(\mathrm{KBr}) 3478,3371,1687 \mathrm{~cm}^{-1} ;{ }^{1} \mathrm{H} \mathrm{NMR}\left(\mathrm{CDCl}_{3}\right) \delta 8.31(\mathrm{~d}, J 8.3$ $\mathrm{Hz}, 1 \mathrm{H}), 8.19$ (d, J 8.4 Hz, 1H), $7.71(\mathrm{t}, J 7.7 \mathrm{~Hz}, 1 \mathrm{H}), 7.62$ (d, J 2.9 Hz, 1H), 7.56 (t, J 7.7 Hz, 1H), $7.13(\mathrm{dd}, J 9.0,2.9 \mathrm{~Hz}, 1 \mathrm{H}), 6.83(\mathrm{~d}, J 9.0 \mathrm{~Hz}, 1 \mathrm{H}), 5.80(\mathrm{br} \mathrm{s}, 2 \mathrm{H}), 3.80(\mathrm{~s}, 3 \mathrm{H}) ;{ }^{13} \mathrm{C} \mathrm{NMR}$ $\delta 166.9,150.7,146.3,145.7,132.7,130.0,126.0,124.9,120.1,118.8,115.8,114.6,112.1,55.9$; MS (EI): $m / z$ [ $\left[\mathrm{M}^{+}\right]$calcd. for $\mathrm{C}_{14} \mathrm{H}_{12} \mathrm{~N}_{4} \mathrm{O}_{2}$ : 268.09, found: 268.0; Anal. Calc. for $\mathrm{C}_{14} \mathrm{H}_{12} \mathrm{~N}_{4} \mathrm{O}$ : C, 62.69; H, 4.48; N, 20.9. Found: C, 62.51; H, 4.61; N, 20.89.

(2-Amino-4-chlorophenyl) (benzotriazole-1-yl)methanone (2g). Yellow solid (1.25 g, 92\%); mp 160-162 ${ }^{\circ}$; IR $v_{\max }(\mathrm{KBr}) 3464,3338,1687 \mathrm{~cm}^{-1} ;{ }^{1} \mathrm{H} \mathrm{NMR}\left(\mathrm{CDCl}_{3}\right) \delta 8.27$ (d, J 8.2 Hz, $1 \mathrm{H}), 8.18(\mathrm{~d}, J 8.2 \mathrm{~Hz}, 1 \mathrm{H}), 8.07(\mathrm{~d}, J 8.7 \mathrm{~Hz}, 1 \mathrm{H}), 7.70(\mathrm{t}, J 7.6 \mathrm{~Hz}, 1 \mathrm{H}), 7.55$ (t, $J .7 \mathrm{~Hz}, 1 \mathrm{H})$, $6.83(\mathrm{~s}, 1 \mathrm{H}), 6.77(\mathrm{~d}, J 8.7 \mathrm{~Hz}, 1 \mathrm{H}), 5.94(\mathrm{br} \mathrm{s}, 2 \mathrm{H}) ;{ }^{13} \mathrm{C}$ NMR $\delta 167.3,153.6,146.6,142.6$, 136.6, 133.4, 130.8, 126.8, 120.9, 117.8, 117.0, 115.2, 110.9; MS (EI): $\mathrm{m} / \mathrm{z}\left[\mathrm{M}^{+}\right]$calcd. for $\mathrm{C}_{13} \mathrm{H}_{9} \mathrm{ClN}_{4} \mathrm{O}: 272.04$, found: 272.0; Anal. Calc. for $\mathrm{C}_{13} \mathrm{H}_{9} \mathrm{ClN}_{4} \mathrm{O}: \mathrm{C}, 57.25 ; \mathrm{H}, 3.33 ; \mathrm{N}, 20.54$. Found: C, 57.28; H, 3.40; N, 20.58.

(2-Amino-5-chlorophenyl) (benzotriazole-1-yl)methanone (2h). Yellow solid (1.01 g, 74\%); mp 153-155 ${ }^{\circ} \mathrm{C}$; IR $v_{\max }(\mathrm{KBr}) 3465,3333,1679 \mathrm{~cm}^{-1} ;{ }^{1} \mathrm{H} \mathrm{NMR}\left(\mathrm{CDCl}_{3}\right) \delta 8.28(\mathrm{~d}, J 8.3 \mathrm{~Hz}$, $1 \mathrm{H}), 8.20(\mathrm{~d}, J 8.3 \mathrm{~Hz}, 1 \mathrm{H}), 8.11(\mathrm{~d}, J 2.3 \mathrm{~Hz}, 1 \mathrm{H}), 7.71(\mathrm{t}, J 7.6 \mathrm{~Hz}, 1 \mathrm{H}), 7.57$ (t, $J 7.7 \mathrm{~Hz}, 1 \mathrm{H})$, $7.36(\mathrm{dd}, J 8.9,2.3,1 \mathrm{H}), 6.78(\mathrm{~d}, J 8.9 \mathrm{~Hz}, 1 \mathrm{H}), 5.83($ br s, $2 \mathrm{H}){ }^{13} \mathrm{C}$ NMR $\delta 167.3,151.3,146.6$, 136.2, 134.0, 133.4, 131.0, 126.9, 121.8, 121.0, 119.1, 115.2, 113.2; MS (EI): $\left.\mathrm{m} / z . \mathrm{M}^{+}\right]$calcd. for $\mathrm{C}_{13} \mathrm{H}_{9} \mathrm{ClN}_{4} \mathrm{O}$ : 272.04, found: 271.9; Anal. Calc. for $\mathrm{C}_{13} \mathrm{H}_{9} \mathrm{ClN}_{4} \mathrm{O}$ : C, 57.25; H, 3.33; N, 20.54 . Found: C, 57.36; H, 3.36; N, 20.33.

(2-Amino-5-bromophenyl) (benzotriazole-1-yl)methanone (2i). Yellow solid (0.840 g, 53\%); mp 151-153 ${ }^{\circ}$; IR $v_{\max }(\mathrm{KBr}) 3465,3330,1677 \mathrm{~cm}^{-1} ;{ }^{1} \mathrm{H} \mathrm{NMR}\left(\mathrm{CDCl}_{3}\right) \delta 8.28(\mathrm{~d}, J 8.3 \mathrm{~Hz}$, $1 \mathrm{H}), 8.24(\mathrm{~d}, J 2.3 \mathrm{~Hz}, 1 \mathrm{H}), 8.20(\mathrm{~d}, J 8.3 \mathrm{~Hz}, 1 \mathrm{H}), 7.72(\mathrm{t}, J 7.6 \mathrm{~Hz}, 1 \mathrm{H}), 7.57$ (t, $J 7.6 \mathrm{~Hz}, 1 \mathrm{H})$, 7.49 (dd, $J 8.9,2.3 \mathrm{~Hz}, 1 \mathrm{H}), 6.73(\mathrm{~d}, J 8.9 \mathrm{~Hz}, 1 \mathrm{H}), 5.83$ (br s, $2 \mathrm{H}) ;{ }^{13} \mathrm{C} \mathrm{NMR} \delta 166.2,150.7$, 145.7, 138.0, 136.1, 132.5, 130.1, 126.2, 120.3, 118.7, 114.5, 113.1, 107.7; MS (EI): $m / z\left[\mathrm{M}^{+}\right]$ calcd. for $\mathrm{C}_{13} \mathrm{H}_{9} \mathrm{BrN}_{4} \mathrm{O}$ : 315.99, found: 315.9; Anal. Calc. for $\mathrm{C}_{13} \mathrm{H}_{9} \mathrm{BrN}_{4} \mathrm{O}$ : C, 49.23; H, 2.86; N, 17.67. Found: C, 49.27; H, 2.95; N, 17.54. 
(2-Amino-5-iodophenyl) (benzotriazole-1-yl)methanone (2j). Yellow solid (1.00 g, 55\%); mp $138-140{ }^{\circ} \mathrm{C}$; IR $v_{\max }(\mathrm{KBr}) 3489,3382,1691 \mathrm{~cm}^{-1} ;{ }^{1} \mathrm{H} \mathrm{NMR}\left(\mathrm{CDCl}_{3}\right) \delta 8.34(\mathrm{~d}, J 2.0 \mathrm{~Hz}, 1 \mathrm{H})$, $8.25(\mathrm{~d}, J 8.3 \mathrm{~Hz}, 1 \mathrm{H}), 8.18(\mathrm{~d}, J 8.3 \mathrm{~Hz}, 1 \mathrm{H}), 7.70(\mathrm{t}, J 7.7 \mathrm{~Hz}, 1 \mathrm{H}), 7.61(\mathrm{dd}, J 8.8,2.0 \mathrm{~Hz}, 1 \mathrm{H})$, $7.55(\mathrm{t}, J 7.7 \mathrm{~Hz}, 1 \mathrm{H}), 6.61(\mathrm{~d}, J 8.8 \mathrm{~Hz}, 1 \mathrm{H}), 5.86(\mathrm{br} \mathrm{s}, 2 \mathrm{H}) ;{ }^{13} \mathrm{C}$ NMR $\delta 166.0,151.2,145.7$, 143.3, 142.0, 132.5, 130.2, 126.2, 120.2, 119.1, 114.5, 113.9, 76.1; MS (EI): $\mathrm{m} / \mathrm{z}\left[\mathrm{M}^{+}\right]$calcd. for $\mathrm{C}_{13} \mathrm{H}_{9} \mathrm{IN}_{4} \mathrm{O}$ : 363.98, found: 363.9; Anal. Calc. for $\mathrm{C}_{13} \mathrm{H}_{9} \mathrm{IN}_{4} \mathrm{O}: \mathrm{C}, 42.88 ; \mathrm{H}, 2.49 ; \mathrm{N}, 15.39$. Found: C, 43.00; H, 2.59; N, 15.17 .

(2-Amino-3, 5-dichlorophenyl) (benzotriazole-1-yl)methanone (2k). Yellow solid (1.43 g, 93\%); mp 170-172 ${ }^{\circ} \mathrm{C}$; IR $v_{\max }(\mathrm{KBr}) 3486,3367,1679 \mathrm{~cm}^{-1}$; ${ }^{1} \mathrm{H} \mathrm{NMR}\left(\mathrm{CDCl}_{3}\right) \delta 8.30(\mathrm{~d}, J 8.2$ $\mathrm{Hz}, 1 \mathrm{H}), 8.21(\mathrm{~d}, J 8.3 \mathrm{~Hz}, 1 \mathrm{H}), 8.07(\mathrm{~d}, J 2.4 \mathrm{~Hz}, 1 \mathrm{H}), 7.74(\mathrm{t}, J 7.7 \mathrm{~Hz}, 1 \mathrm{H}), 7.60-7.57(\mathrm{~m}, 2 \mathrm{H})$, 6.26 (br s, $2 \mathrm{H}$ ); ${ }^{13} \mathrm{C}$ NMR $\delta 166.0,146.4,145.8,134.5,132.5,132.2,130.4,126.4,121.2,120.4$, 120.3, 114.6, 113.3; MS (EI): $\mathrm{m} / z$ [ $\left.\mathrm{M}^{+}\right]$calcd. for $\mathrm{C}_{13} \mathrm{H}_{8} \mathrm{Cl}_{2} \mathrm{~N}_{4} \mathrm{O}$ : 306.00, found: 305.9; Anal. Calc. for $\mathrm{C}_{13} \mathrm{H}_{8} \mathrm{Cl}_{2} \mathrm{~N}_{4} \mathrm{O}$ : C, 50.65; H, 2.62; N, 18.18. Found: C, 50.87; H, 2.76; N, 17.86 .

(2-Amino-3, 5-dibromophenyl) (benzotriazole-1-yl)methanone (2l). Yellow solid (1.64 g, 83\%); mp 174-176 ${ }^{\circ} \mathrm{C}$; IR $v_{\max }(\mathrm{KBr}) 3488,3366,1608 \mathrm{~cm}^{-1}$; ${ }^{1} \mathrm{H} \mathrm{NMR}\left(\mathrm{CDCl}_{3}\right) \delta 8.29(\mathrm{~d}, J 8.3$ $\mathrm{Hz}, 1 \mathrm{H}), 8.22-8.20(\mathrm{~m}, 2 \mathrm{H}), 7.85(\mathrm{~d}, J 2.2 \mathrm{~Hz}, 1 \mathrm{H}), 7.74(\mathrm{t}, J 7.7 \mathrm{~Hz}, 1 \mathrm{H}), 7.59(\mathrm{t}, J 7.7 \mathrm{~Hz}$, 1H), 6.32 (br s, 2H); ${ }^{13} \mathrm{C}$ NMR $\delta 165.8,147.5,145.8,140.1,135.8,132.5,130.4,126.4,120.4$,

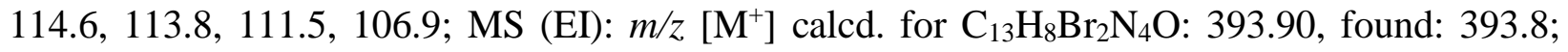
Anal. Calc. for $\mathrm{C}_{13} \mathrm{H}_{8} \mathrm{Br}_{2} \mathrm{~N}_{4} \mathrm{O}: \mathrm{C}, 39.32 ; \mathrm{H}, 2.03 ; \mathrm{N}, 14.11$. Found: C, 39.56; H, 2.19; N, 13.78 .

(2-Amino-3, 5-diiodophenyl) (benzotriazole-1-yl)methanone (2m). Yellow solid (1.57 g, 64\%); mp decomposed $>220{ }^{\circ} \mathrm{C}$; IR $v_{\max }(\mathrm{KBr}) 3489,3375,1667 \mathrm{~cm}^{-1} ;{ }^{1} \mathrm{H} \mathrm{NMR}\left(\mathrm{CDCl}_{3}\right) \delta 8.30$ - $8.19(\mathrm{~m}, 4 \mathrm{H}), 7.73(\mathrm{t}, J 7.7 \mathrm{~Hz}, 1 \mathrm{H}), 7.59(\mathrm{t}, J 7.7 \mathrm{~Hz}, 1 \mathrm{H}), 6.35(\mathrm{br} \mathrm{s}, 2 \mathrm{H}) ;{ }^{13} \mathrm{C}$ NMR $\delta 165.8$, 151.6, 149.9, 145.8, 142.6, 132.5, 130.4, 126.4, 120.4, 114.5, 113.8, 87.3, 75.9; MS (EI): $\mathrm{m} / \mathrm{z}$ $\left[\mathrm{M}^{+}\right]$calcd. for $\mathrm{C}_{13} \mathrm{H}_{8} \mathrm{I}_{2} \mathrm{~N}_{4} \mathrm{O}: 489.87$, found: 489.7; Anal. Calc. for $\mathrm{C}_{13} \mathrm{H}_{8} \mathrm{I}_{2} \mathrm{~N}_{4} \mathrm{O}: \mathrm{C}, 31.80 ; \mathrm{H}$, 1.64; N, 11.41. Found: C, 32.00; H, 1.78; N, 11.33.

(2-Amino-4, 5-dimethoxyphenyl) (benzotriazole-1-yl)methanone (2n). Yellow solid (1.04 g, 70\%); mp 93-95 ${ }^{\circ} \mathrm{C}$; IR $v_{\max }(\mathrm{KBr}) 3470,3361,1672 \mathrm{~cm}^{-1} ;{ }^{1} \mathrm{H}$ NMR $\left(\mathrm{CDCl}_{3}\right) \delta 8.25(\mathrm{~d}, J 8.3 \mathrm{~Hz}$, $1 \mathrm{H}), 8.17(\mathrm{~d}, J 8.3 \mathrm{~Hz}, 1 \mathrm{H}), 7.72(\mathrm{~s}, 1 \mathrm{H}), 7.68(\mathrm{t}, J 7.7 \mathrm{~Hz}, 1 \mathrm{H}), 7.53(\mathrm{t}, J 7.6 \mathrm{~Hz}, 1 \mathrm{H}), 6.27$ (s, $1 \mathrm{H}), 6.05$ (br s, 2H), 3.96 (s, 3H), $3.84(\mathrm{~s}, 3 \mathrm{H}) ;{ }^{13} \mathrm{C}$ NMR $\delta 165.9,156.6,150.6,145.5,140.8$, 133.0, 129.6, 125.7, 120.0, 115.0, 114.5, 102.7, 99.0, 56.4, 56.0; MS (EI): $\mathrm{m} / z\left[\mathrm{M}^{+}\right]$calcd. for $\mathrm{C}_{15} \mathrm{H}_{14} \mathrm{~N}_{4} \mathrm{O}_{3}$ : 298.10, found: 298.0; Anal. Calc. for $\mathrm{C}_{15} \mathrm{H}_{14} \mathrm{~N}_{4} \mathrm{O}_{3}: \mathrm{C}, 60.39 ; \mathrm{H}, 4.73 ; \mathrm{N}, 18.78$. Found: C, 60.45; H, 4.79; N, 18.89 .

(2-Amino-3, 4, 5-trimethoxyphenyl) (benzotriazole-1-yl)methanone (2o). Yellow solid (1.23 g, 75\%); mp 115-117 ${ }^{\circ} \mathrm{C}$; IR $v_{\max }(\mathrm{KBr}) 3465,3342,1664 \mathrm{~cm}^{-1} ;{ }^{1} \mathrm{H} \mathrm{NMR}\left(\mathrm{CDCl}_{3}\right) \delta 8.28(\mathrm{~d}, J$ $8.3 \mathrm{~Hz}, 1 \mathrm{H}), 8.18(\mathrm{~d}, J 8.3 \mathrm{~Hz}, 1 \mathrm{H}), 7.69(\mathrm{t}, J 7.6 \mathrm{~Hz}, 1 \mathrm{H}), 7.55(\mathrm{t}, J 7.6 \mathrm{~Hz}, 1 \mathrm{H}), 7.52(\mathrm{~s}, 1 \mathrm{H})$, 6.08 (br s, 2H), 4.07 (s, 3H), 3.95 (s, 3H), $3.82(\mathrm{~s}, 3 \mathrm{H}) ;{ }^{13} \mathrm{C}$ NMR $\delta$ 166.3, 148.8, 145.6, 144.0, 143.5, 139.9, 132.9, 129.8, 125.8, 120.0, 114.6, 111.1, 105.2, 61.0, 60.5, 56.4; MS (EI): $\mathrm{m} / \mathrm{z}\left[\mathrm{M}^{+}\right]$ calcd. for $\mathrm{C}_{16} \mathrm{H}_{16} \mathrm{~N}_{4} \mathrm{O}_{4}: 328.11$, found: 328.0; Anal. Calc. for $\mathrm{C}_{16} \mathrm{H}_{16} \mathrm{~N}_{4} \mathrm{O}_{4}: \mathrm{C}, 58.53 ; \mathrm{H}, 4.91 ; \mathrm{N}$, 17.07. Found: C, 58.70; H, 4.93; N, 17.24. 
General experimental procedure for the preparation of primary anthranilamides (3a-g). $N$ (2-Aminoarylacyl)benzotriazoles $(1 \mathrm{mmol})$ were treated with excess ammonium hydroxide (10 $\mathrm{mmol})$ in THF $(10 \mathrm{~mL})$ cooled in an ice bath for 2 hours. After the completion of the reaction, the solvent was removed under reduced pressure and then the residue was purified by column chromatography over slica gel with EtOAc/ n-Hexane (1:1) to give the desired primary amides as white solids.

2-Aminobenzamide (3a). White solid (0.130 g, 96\%); mp112-113 ${ }^{\circ} \mathrm{C}$; IR $v_{\max }(\mathrm{KBr}) 3409.5$, 3316.0, 3201.3, 1660.4 $\mathrm{cm}^{-1}$; ${ }^{1} \mathrm{H}$ NMR (DMSO-d 6 ) $\delta 7.74$ (br s, 1H), 7.52 (d, J 7.9 Hz, 1H), 7.13 (t, J 7.6 Hz, 1H), 7.06 (br s, 1H), 6.67 (d, J 8.2 Hz, 1H), 6.55 (br s, 2H), 6.48 (t, J 7.5 Hz, 1H); ${ }^{13} \mathrm{C}$ NMR $\delta 172.8,151.5,133.2,130.0,117.6,115.6,114.8 ; \mathrm{MS}(\mathrm{EI}): \mathrm{m} / z\left[\mathrm{M}^{+}\right]$calcd. for $\mathrm{C}_{7} \mathrm{H}_{8} \mathrm{~N}_{2} \mathrm{O}$ : 136.06, found: 136.0; Anal. Calc. for $\mathrm{C}_{7} \mathrm{H}_{8} \mathrm{~N}_{2} \mathrm{O}$ : C, 61.75; H, 5.92; N, 20.58. Found: C, 61.95; H, 6.28; N, 20.20.

2-Amino-4-methylbenzamide (3b). White solid (0.140 g, 93\%); mp148-149 ${ }^{\circ} \mathrm{C}$; IR $v_{\max }(\mathrm{KBr})$ 3409.5, 3294.8, 3172.3, $1674.9 \mathrm{~cm}^{-1} ;{ }^{1} \mathrm{H}$ NMR (DMSO- $\left.d_{6}\right) \delta 7.65$ (br s, $\left.1 \mathrm{H}\right), 7.43$ (d, $J 8.1 \mathrm{~Hz}$, 1H), 6.95 (br s, 1H), 6.54 (br s, 2H), $6.48(\mathrm{~s}, 1 \mathrm{H}), 6.30$ (d, J 8.0 Hz, 1H), 2.16 (s, 3H); ${ }^{13} \mathrm{C} \mathrm{NMR}$ $\delta 172.8,151.7,143.0,130.0,117.7,116.9,112.2,21.7 ; \mathrm{MS}(\mathrm{EI}): m / z\left[\mathrm{M}^{+}\right]$calcd. for $\mathrm{C}_{8} \mathrm{H}_{10} \mathrm{~N}_{2} \mathrm{O}$ : 150.07, found: 150.0; Anal. Calc. for $\mathrm{C}_{8} \mathrm{H}_{10} \mathrm{~N}_{2} \mathrm{O}$ : C, 63.98; H, 7.19; N, 18.64. Found: C, 64.21; H, 7.19; N, 18.20.

2-Amino-4-chlorobenzamide (3c). White solid (0.150 g, 88\%); mp180-182 ${ }^{\circ} \mathrm{C}$; IR $v_{\max }(\mathrm{KBr})$ 3496.3, 3351.7, 3164.6, 3085.5, $1667.2 \mathrm{~cm}^{-1} ;{ }^{1} \mathrm{H}$ NMR (DMSO- $\left.d_{6}\right) \delta 7.80$ (br s, 1H), $7.54(\mathrm{~d}, J$ $8.4 \mathrm{~Hz}, 1 \mathrm{H}), 7.16$ (br s, 1H), 6.83 (br s, 2H), $6.74(\mathrm{~s}, 1 \mathrm{H}), 6.50(\mathrm{t}, J 8.4 \mathrm{~Hz}, 1 \mathrm{H}) ;{ }^{13} \mathrm{C} \mathrm{NMR} \delta$ 172.0, 152.8, 137.6, 131.8, 116.3, 115.2, 113.5; $\mathrm{MS}(\mathrm{EI}): \mathrm{m} / z\left[\mathrm{M}^{+}\right]$calcd. for $\mathrm{C}_{7} \mathrm{H}_{7} \mathrm{ClN}_{2} \mathrm{O}$ : 170.02, found: 170.0; Anal. Calc. for $\mathrm{C}_{7} \mathrm{H}_{7} \mathrm{ClN}_{2} \mathrm{O}$ : C, 49.27; H, 4.13; N, 16.42. Found: C, 49.18; H, 4.48; N, 16.06 .

2-Amino-5-bromobenzamide (3d). White solid (0.200 g, 93\%); mp 185-187 ${ }^{\circ} \mathrm{C}$; IR $v_{\max }(\mathrm{KBr})$ 3395.1, 3287.1, 3164.6, 1674.9 $\mathrm{cm}^{-1}$; ${ }^{1} \mathrm{H}$ NMR (DMSO- $\left.d_{6}\right) \delta 7.86$ (br s, 1H), 7.70 (d, J $2.5 \mathrm{~Hz}$, $1 \mathrm{H}), 7.26(\mathrm{dd}, J 9.0,2.0 \mathrm{~Hz}, 1 \mathrm{H}), 7.19(\mathrm{br} \mathrm{s}, 1 \mathrm{H}), 6.71($ br s, $2 \mathrm{H}), 6.66(\mathrm{~d}, J 8.5 \mathrm{~Hz}, 1 \mathrm{H}) ;{ }^{13} \mathrm{C}$ NMR $\delta 170.5,149.8,134.8,131.2,119.0,115.6,105.2 ; \mathrm{MS}(\mathrm{EI}): \mathrm{m} / \mathrm{z}\left[\mathrm{M}^{+}\right]$calcd. for $\mathrm{C}_{7} \mathrm{H}_{7} \mathrm{BrN}_{2} \mathrm{O}$ : 213.97, found: 214.0; Anal. Calc. for $\mathrm{C}_{7} \mathrm{H}_{7} \mathrm{BrN}_{2} \mathrm{O}$ : C, 39.09; H, 3.28; N, 13.03 . Found: C, 39.40; H, 3.57; N, 12.88 .

2-Amino-5-iodobenzamide (3e). White solid (0.232 g, 89\%); mp 177-178 ${ }^{\circ} \mathrm{C}$; IR $v_{\max }(\mathrm{KBr})$ 3395.1, 3287.1, 3172.3, $1674.9 \mathrm{~cm}^{-1}$; ${ }^{1} \mathrm{H}$ NMR (DMSO- $\left.d_{6}\right) \delta 7.85$ (br s, $\left.1 \mathrm{H}\right), 7.81$ (d, $J 1.5 \mathrm{~Hz}$, 1H), 7.38 (dd, J 9.0, $2.0 \mathrm{~Hz}, 1 \mathrm{H}), 7.16$ (br s, 1H), 6.70 (br s, $2 \mathrm{H}), 6.55(\mathrm{~d}, J 8.5 \mathrm{~Hz}, 1 \mathrm{H}) ;{ }^{13} \mathrm{C}$ NMR $\delta 171.4,151.1,141.2,137.8,120.2,117.3,75.4 ; \mathrm{MS}(\mathrm{EI}): m / z\left[\mathrm{M}^{+}\right]$calcd. for $\mathrm{C}_{7} \mathrm{H}_{7} \mathrm{IN}_{2} \mathrm{O}$ : 261.95, found: 262.0; Anal. Calc. for $\mathrm{C}_{7} \mathrm{H}_{7} \mathrm{IN}_{2} \mathrm{O}$ : C, 32.08; H, 2.69; N, 10.69. Found: C, 32.45; $\mathrm{H}, 3.01 ; \mathrm{N}, 10.36$.

2-Amino-4,5-dimethoxybenzamide (3f). White solid $(0.160 \mathrm{~g}, 82 \%)$; mp $148-149^{\circ} \mathrm{C}$; IR $v_{\max }$ (KBr) 3438.5, 3337.2, $3238.0 \mathrm{~cm}^{-1}, 1681.6 ;{ }^{1} \mathrm{H}$ NMR (DMSO-d 6 ) $\delta 7.58$ (br s, 1H), $7.12-7.09$ $(\mathrm{m}, 1 \mathrm{H}), 6.82$ (br s, 1H), 6.44 (br s, 2H), $6.30-6.26(\mathrm{~m}, 1 \mathrm{H}), 3.72(\mathrm{~s}, 3 \mathrm{H}), 3.67(\mathrm{~s}, 3 \mathrm{H}) ;{ }^{13} \mathrm{C}$ NMR $\delta 172.4,154.3,148.1,140.2,114.1,105.3,100.8,57.4,55.9 ; \mathrm{MS}(\mathrm{EI}): \mathrm{m} / z\left[\mathrm{M}^{+}\right]$calcd. for 
$\mathrm{C}_{9} \mathrm{H}_{12} \mathrm{~N}_{2} \mathrm{O}_{3}$ : 196.08, found: 196.0; Anal. Calc. for $\mathrm{C}_{9} \mathrm{H}_{12} \mathrm{~N}_{2} \mathrm{O}_{3}$ : C, 55.09; H, 6.17; N, 14.28. Found: C, 55.28; H, 6.54; N, 13.92 .

2-Amino-3,5-dibromobenzamide (3g). White solid $(0.250 \mathrm{~g}, 85 \%)$; mp 214-215 ${ }^{\circ} \mathrm{C}$; IR $v_{\max }$ (KBr) 3366.1, 3322.7, 3179.1, $1652.7 \mathrm{~cm}^{-1}$; ${ }^{1} \mathrm{H}$ NMR (DMSO-d6) $\delta 8.06$ (br s, 1H), $7.79-7.76$ $(\mathrm{m}, 1 \mathrm{H}), 7.74-7.72(\mathrm{~m}, 1 \mathrm{H}), 7.47$ (br s, $1 \mathrm{H}), 6.80$ (br s, $2 \mathrm{H}) ;{ }^{13} \mathrm{C}$ NMR $\delta 170.7,147.3,137.9$, 131.9, 118.0, 111.5, 106.0; $\mathrm{MS}$ (EI): $\mathrm{m} / z$ [ $\left[\mathrm{M}^{+}\right]$calcd. for $\mathrm{C}_{7} \mathrm{H}_{6} \mathrm{Br}_{2} \mathrm{~N}_{2} \mathrm{O}$ : 291.88, found: 291.9; Anal. Calc. for $\mathrm{C}_{7} \mathrm{H}_{6} \mathrm{Br}_{2} \mathrm{~N}_{2} \mathrm{O}$ : C, 28.60; H, 2.06; N, 9.53. Found: C, 28.85; H, 2.43; N, 9.23.

General experimental procedure for the preparation of secondary (4a-i) and tertiary athranilamides (5a-h). $N$-(2-Aminoarylacyl)benzotriazoles $(1 \mathrm{mmol})$ and various primary and secondary amines $(1.2 \mathrm{mmol})$ were left to stir in $\mathrm{CH}_{2} \mathrm{Cl}_{2}(10 \mathrm{~mL})$ for 2-6 hours at room temperature. After the reaction ended, the solvent was removed under reduced pressure. The residue was purified by column chromatography over slica gel with EtOAc/ n-hexane prepared in different proportions to afford secondary and tertiary amides.

2-Amino- $N$-benzylbenzamide (4a). White solid $(0.160 \mathrm{~g}, 71 \%)$; mp $121-122{ }^{\circ} \mathrm{C}$; IR $v_{\max }(\mathrm{KBr})$ 3467.4, 3359.4, 3301.5, $1638.2 \mathrm{~cm}^{-1} ;{ }^{1} \mathrm{H}$ NMR $\left(\mathrm{CDCl}_{3}\right) \delta 7.41-7.38(\mathrm{~m}, 4 \mathrm{H}), 7.36-7.33(\mathrm{~m}$, 2H), $7.26-7.22(\mathrm{~m}, 1 \mathrm{H}), 6.72(\mathrm{~d}, J 8.2 \mathrm{~Hz}, 1 \mathrm{H}), 6.66(\mathrm{t}, J 7.5 \mathrm{~Hz}, 1 \mathrm{H}), 6.35$ (br s, 1H), 5.62 (br s, 2H), 4.64 (d, J 5.6 Hz, 2H); ${ }^{13} \mathrm{C}$ NMR $\delta 169.2,148.8,138.3,132.4,128.8,127.8,127.6,127.1$, 117.4, 116.7, 115.9, 43.8; MS (EI): $m / z\left[\mathrm{M}^{+}\right]$calcd. for $\mathrm{C}_{14} \mathrm{H}_{14} \mathrm{~N}_{2} \mathrm{O}: 226.11$, found: 226.1; Anal. Calc. for $\mathrm{C}_{14} \mathrm{H}_{14} \mathrm{~N}_{2} \mathrm{O}: \mathrm{C}, 74.31 ; \mathrm{H}, 6.24 ; \mathrm{N}, 12.38$. Found: C, 74.02; H, 6.37; N, 12.09 .

2-Amino-3-methyl- $\boldsymbol{N}$-propylbenzamide (4b). White solid $(0.152 \mathrm{~g}, 79 \%)$; mp 105-106 ${ }^{\circ} \mathrm{C}$; IR $v_{\max }(\mathrm{KBr}) 3424.0,3301.5,1623.8 \mathrm{~cm}^{-1} ;{ }^{1} \mathrm{H} \mathrm{NMR}\left(\mathrm{CDCl}_{3}\right) \delta 7.23(\mathrm{~d}, J 7.8 \mathrm{~Hz}, 1 \mathrm{H}), 7.15(\mathrm{~d}, J 7.3$ $\mathrm{Hz}, 1 \mathrm{H}), 6.62$ (t, J 7.6 Hz, 1H), 6.10 (br s, 1H), 5.63 (br s, 2H), 3.40 (q, J $6.7 \mathrm{~Hz}, 2 \mathrm{H}$ ), 2.19 (s, $3 \mathrm{H}), 1.65(\mathrm{sex}, J 7.3 \mathrm{~Hz}, 2 \mathrm{H}), 1.01(\mathrm{t}, J 7.4 \mathrm{~Hz}, 3 \mathrm{H}) ;{ }^{13} \mathrm{C} \mathrm{NMR} \delta 170.9,147.7,133.8,125.6$, 124.6, 116.8, 41.7, 23.1, 17.6, 11.6; MS (EI): $m / z\left[\mathrm{M}^{+}\right]$calcd. for $\mathrm{C}_{11} \mathrm{H}_{16} \mathrm{~N}_{2} \mathrm{O}$ : 192.12, found: 192.1; Anal. Calc. for $\mathrm{C}_{11} \mathrm{H}_{16} \mathrm{~N}_{2} \mathrm{O}$ : C, 68.72; H, 8.39; N, 14.57. Found: C, 68.68; H, 8.36; N, 14.36.

2-Amino- $N$-cyclohexyl-4-methylbenzamide (4c). White solid $(0.200 \mathrm{~g}, 86 \%)$; mp 150-151 ${ }^{\circ} \mathrm{C}$; IR $v_{\max }(\mathrm{KBr}) 3474.1,3359.4,3301.5,1638.2 \mathrm{~cm}^{-1} ;{ }^{1} \mathrm{H} \mathrm{NMR}\left(\mathrm{CDCl}_{3}\right) \delta 7.20(\mathrm{~d}, J 8.0 \mathrm{~Hz}, 1 \mathrm{H})$, $6.52(\mathrm{~s}, 1 \mathrm{H}), 6.49(\mathrm{~d}, J 8.0 \mathrm{~Hz}, 1 \mathrm{H}), 5.89$ (d, J $6.5 \mathrm{~Hz}, 1 \mathrm{H}), 5.57$ (br s, 2H), $3.97-3.91$ (m, 1H), $2.25(\mathrm{~s}, 3 \mathrm{H}), 2.05-2.01(\mathrm{~m}, 2 \mathrm{H}), 1.79-1.75(\mathrm{~m}, 2 \mathrm{H}), 1.69-1.65(\mathrm{~m}, 1 \mathrm{H}), 1.48-1.40(\mathrm{~m}, 2 \mathrm{H})$, $1.20-1.28(\mathrm{~m}, 3 \mathrm{H}) ;{ }^{13} \mathrm{C} \mathrm{NMR} \delta 168.4,148.6,142.6,126.9,117.9,117.7,114.0,48.2,33.3$, 25.6, 24.9, 21.4; MS (EI): $\mathrm{m} / z\left[\mathrm{M}^{+}\right]$calcd. for $\mathrm{C}_{14} \mathrm{H}_{20} \mathrm{~N}_{2} \mathrm{O}: 232.15$, found: 232.1; Anal. Calc. for $\mathrm{C}_{14} \mathrm{H}_{20} \mathrm{~N}_{2} \mathrm{O}$ : C, 72.38; H, 8.68; N, 12.06. Found: C, 72.01; H, 8.69; N, 11.86.

2-Amino- $N$-cyclohexyl-5-methylbenzamide (4d). White solid $(0.200 \mathrm{~g}, 86 \%)$; mp 201-202 ${ }^{\circ} \mathrm{C}$; IR $v_{\max }(\mathrm{KBr}) 3417.2,3294.8,1631.5 \mathrm{~cm}^{-1} ;{ }^{1} \mathrm{H} \mathrm{NMR}\left(\mathrm{CDCl}_{3}\right) \delta 7.11(\mathrm{~s}, 1 \mathrm{H}), 7.06(\mathrm{~d}, J 8.8 \mathrm{~Hz}$, $1 \mathrm{H}), 6.68(\mathrm{~d}, J 8.5 \mathrm{~Hz}, 1 \mathrm{H}), 5.96$ (br s, 1H), 5.40 (br s, 2H), $3.95-3.93(\mathrm{~m}, 1 \mathrm{H}), 2.29-2.24(\mathrm{~m}$, $2 \mathrm{H}), 2.05-2.02(\mathrm{~m}, 2 \mathrm{H}), 1.79-1.76(\mathrm{~m}, 2 \mathrm{H}), 1.69-1.66(\mathrm{~m}, 1 \mathrm{H}), 1.46-1.41(\mathrm{~m}, 2 \mathrm{H}), 1.30-$ $1.22(\mathrm{~m}, 4 \mathrm{H}) ;{ }^{13} \mathrm{C}$ NMR $\delta 169.4,146.2,133.7,127.9,127.2,118.6,118.0,48.6,33.5,25.8,25.1$, 
20.6; MS (EI): $m / z$ [ $\left[\mathrm{M}^{+}\right]$calcd. for $\mathrm{C}_{14} \mathrm{H}_{20} \mathrm{~N}_{2} \mathrm{O}: 232.15$, found: 232.1; Anal. Calc. for $\mathrm{C}_{14} \mathrm{H}_{20} \mathrm{~N}_{2} \mathrm{O}$ : C, 72.38; H, 8.68; N, 12.06. Found: C, 72.15; H, 8.85; N, 11.84 .

2-Amino-5-methoxy- $\boldsymbol{N}$-propylbenzamide (4e). White solid $(0.171 \mathrm{~g}, 82 \%)$; mp $72-74{ }^{\circ} \mathrm{C}$; IR $v_{\max }(\mathrm{KBr}) 3413.4,3301.5,1654.6 \mathrm{~cm}^{-1} ;{ }^{1} \mathrm{H}$ NMR $\left(\mathrm{CDCl}_{3}\right) \delta 6.90-6.87(\mathrm{~m}, 2 \mathrm{H}), 6.68(\mathrm{~d}, J 8.5$ $\mathrm{Hz}, 1 \mathrm{H}), 6.22$ (br s, 1H), 5.01 (br s, 2H), 3.77 (s, 3H), 3.38 (q, J 6.7 Hz, 2H), 1.64 (sex, J 7.2 Hz, 2H), $0.99(\mathrm{t}, J 7.5 \mathrm{~Hz}, 3 \mathrm{H}) ;{ }^{13} \mathrm{C} \mathrm{NMR} \delta 169.0,151.3,142.0,118.8,118.7,118.1,112.3,56.1$, 41.5, 23.0, 11.5; MS (EI): $m / z\left[\mathrm{M}^{+}\right]$calcd. for $\mathrm{C}_{11} \mathrm{H}_{16} \mathrm{~N}_{2} \mathrm{O}_{2}$ : 208.12, found: 208.1; Anal. Calc. for $\mathrm{C}_{11} \mathrm{H}_{16} \mathrm{~N}_{2} \mathrm{O}_{2}$ : C, 63.44; H, 7.74; N, 13.45. Found: C, 63.48; H, 7.86; N, 13.74.

2-Amino-4-chloro- $\boldsymbol{N}$-methylbenzamide (4f). White solid $\left(0.172 \mathrm{~g}\right.$, 93\%); mp 96-97 ${ }^{\circ} \mathrm{C}$; IR $v_{\max }$ (KBr) 3467.4, 3366.1, 3337.2, 1638.2 cm ${ }^{-1} ;{ }^{1} \mathrm{H}$ NMR $\left(\mathrm{CDCl}_{3}\right) \delta 7.24$ (d, $\left.J 8.4 \mathrm{~Hz}, 1 \mathrm{H}\right), 6.69$ (d, $J$ $2.0 \mathrm{~Hz}, 1 \mathrm{H}), 6.60$ (dd, J 8.4, $2.0 \mathrm{~Hz}, 1 \mathrm{H}), 6.19$ (br s, 1H), 5.66 (br s, 2H), 2.96 (d, J 5.2 Hz, 3H); ${ }^{13} \mathrm{C}$ NMR $\delta 169.3,149.7,137.9,128.4,116.6,116.5,114.5,26.6$; MS (EI): $\mathrm{m} / z\left[\mathrm{M}^{+}\right]$calcd. for $\mathrm{C}_{8} \mathrm{H}_{9} \mathrm{ClN}_{2} \mathrm{O}$ : 184.03, found: 184.0; Anal. Calc. for $\mathrm{C}_{8} \mathrm{H}_{9} \mathrm{ClN}_{2} \mathrm{O}: \mathrm{C}, 52.03 ; \mathrm{H}, 4.91 ; \mathrm{N}, 15.17$. Found: C, 52.34; H, 5.22; N, 14.86 .

2-Amino-5-bromo- $\boldsymbol{N}$-isopropylbenzamide (4g). White solid $(0.224 \mathrm{~g}, 87 \%)$; mp $188-189{ }^{\circ} \mathrm{C}$; IR $v_{\max }(\mathrm{KBr}) 3467.4,3366.1,3287.1,1631.5 \mathrm{~cm}^{-1} ;{ }^{1} \mathrm{H} \mathrm{NMR}\left(\mathrm{CDCl}_{3}\right) \delta 7.40(\mathrm{~d}, J 2.2 \mathrm{~Hz}, 1 \mathrm{H})$, 7.29 (dd, $J$ 8.5, 2.4 Hz, 1H), 6.59 (d, J 8.7 Hz, 1H), 5.82 (br s, 1H), 5.53 (br s, 2H), 4.24 (sep, $J$ $6.6 \mathrm{~Hz}, 1 \mathrm{H}), 1.28(\mathrm{~d}, J 6.6 \mathrm{~Hz}, 6 \mathrm{H}) ;{ }^{13} \mathrm{C} \mathrm{NMR} \delta 168.3,148.5,135.5,130.3,119.6,118.8,108.3$, 42.0, 23.0; $\mathrm{MS}$ (EI): $\mathrm{m} / z\left[\mathrm{M}^{+}\right]$calcd. for $\mathrm{C}_{10} \mathrm{H}_{13} \mathrm{BrN}_{2} \mathrm{O}$ : 256.02, found: 256.0; Anal. Calc. for $\mathrm{C}_{10} \mathrm{H}_{13} \mathrm{BrN}_{2} \mathrm{O}: \mathrm{C}, 46.71 ; \mathrm{H}, 5.10 ; \mathrm{N}, 10.89$. Found: C, 46.69; H, 5.43; N, 10.55.

2-Amino-3,5-dichloro- $N$-propylbenzamide (4h). White solid $(0.234 \mathrm{~g}, 95 \%)$; mp 143-144 ${ }^{\circ} \mathrm{C}$; IR $v_{\max }(\mathrm{KBr}) 3430.7,3287.1,1638.2 \mathrm{~cm}^{-1} ;{ }^{1} \mathrm{H}$ NMR $\left(\mathrm{CDCl}_{3}\right) \delta 7.36(\mathrm{~d}, J 2.3 \mathrm{~Hz}, 1 \mathrm{H}), 7.24(\mathrm{~d}, J$ $2.3 \mathrm{~Hz}, 1 \mathrm{H}$ ), 6.06 (br s, 1H), 5.98 (br s, 2H), 3.39 (q, J $6.7 \mathrm{~Hz}, 2 \mathrm{H}), 1.66$ (sex, J 7.3 Hz, 2H), $1.01(\mathrm{t}, J 7.4 \mathrm{~Hz}, 3 \mathrm{H}) ;{ }^{13} \mathrm{C}$ NMR $\delta 168.6,144.5,132.3,126.1,122.0,120.8,118.8,41.9,23.0$, 11.5; MS (EI): $m / z\left[\mathrm{M}^{+}\right]$calcd. for $\mathrm{C}_{10} \mathrm{H}_{12} \mathrm{Cl}_{2} \mathrm{~N}_{2} \mathrm{O}$ : 246.03, found: 246.0; Anal. Calc. for $\mathrm{C}_{10} \mathrm{H}_{12} \mathrm{Cl}_{2} \mathrm{~N}_{2} \mathrm{O}$ : C, 48.58; H, 4.89; N, 11.33. Found: C, 48.82; H, 5.23; N, 11.07.

2-Amino-4,5-dimethoxy- $\boldsymbol{N}$-methylbenzamide (4i). White solid (0.176 g, 84\%); mp 117-119 ${ }^{\circ} \mathrm{C}$; IR $v_{\max }(\mathrm{KBr}) 3455.8,3390.2,3336.2,1631.5 \mathrm{~cm}^{-1} ;{ }_{1}^{1} \mathrm{H} \mathrm{NMR}\left(\mathrm{CDCl}_{3}\right) \delta 6.84(\mathrm{~s}, 1 \mathrm{H}), 6.20(\mathrm{~s}$, 1H), 6.19 (br s, 1H), 5.40 (br s, 2H), 3.85 (s, 3H), 3.80 (s, 3H) 2.94 (d, J 4.5 Hz, 3H); ${ }^{13} \mathrm{C} \mathrm{NMR} \delta$ 169.7, 153.1, 144.6, 140.8, 110.9, 107.3, 100.8, 56.9, 55.7, 26.5; MS (EI): $m / z\left[\mathrm{M}^{+}\right]$calcd. for $\mathrm{C}_{10} \mathrm{H}_{14} \mathrm{~N}_{2} \mathrm{O}_{3}$ : 210.09, found: 210.1; Anal. Calc. for $\mathrm{C}_{10} \mathrm{H}_{14} \mathrm{~N}_{2} \mathrm{O}_{3}: \mathrm{C}, 57.13 ; \mathrm{H}, 6.71$; N, 13.33 . Found: C, 57.08; H, 6.66; N, 13.61.

(2-Aminophenyl)(piperidin-1-yl)methanone (5a). White solid (0.184 g, 90\%); mp 74-76 ${ }^{\circ} \mathrm{C}$; IR $v_{\max }(\mathrm{KBr}) 3488.5,3359.4,3251.4,1652.7 \mathrm{~cm}^{-1} ;{ }^{1} \mathrm{H} \mathrm{NMR}\left(\mathrm{CDCl}_{3}\right) \delta 7.15(\mathrm{t}, J 7.7 \mathrm{~Hz}, 1 \mathrm{H})$, $7.07(\mathrm{dd}, J 7.5,1.5 \mathrm{~Hz}, 1 \mathrm{H}), 6.72-6.69(\mathrm{~m}, 2 \mathrm{H}), 4.28(\mathrm{~s}, 2 \mathrm{H}), 3.55(\mathrm{br} \mathrm{s}, 4 \mathrm{H}), 1.68-1.60(\mathrm{~m}$, $6 \mathrm{H}) ;{ }^{13} \mathrm{C}$ NMR $\delta 169.7,145.4,130.3,127.6,120.5,117.4,116.6,26.4,26.2,24.6$; MS (EI): $\mathrm{m} / z$ [M $\mathrm{M}^{+}$] calcd. for $\mathrm{C}_{12} \mathrm{H}_{16} \mathrm{~N}_{2} \mathrm{O}$ : 204.12, found: 204.1; Anal. Calc. for $\mathrm{C}_{12} \mathrm{H}_{16} \mathrm{~N}_{2} \mathrm{O}$ : C, 70.56; H, 7.90; N, 13.71. Found: C, 76.56; H, 8.22; N, 13.77 .

(2-Amino-3-methylphenyl)(piperidin-1-yl)methanone (5b). White solid (0.188 g, 86\%); mp 80-81 ${ }^{\circ} \mathrm{C}$; IR $v_{\max }(\mathrm{KBr}) 3430.7,3351.7,1617.0 \mathrm{~cm}^{-1} ;{ }^{1} \mathrm{H} \mathrm{NMR}\left(\mathrm{CDCl}_{3}\right) \delta 7.05(\mathrm{~s}, J 7.5 \mathrm{~Hz}, 1 \mathrm{H})$, 
$6.95(\mathrm{~s}, J 7.5 \mathrm{~Hz}, 1 \mathrm{H}), 6.64(\mathrm{t}, J 7.5 \mathrm{~Hz}, 1 \mathrm{H}), 4.25(\mathrm{~s}, 2 \mathrm{H}), 3.55$ (br s, 4H), 2.17 (s, 3H), 1.67 $1.59(\mathrm{~m}, 6 \mathrm{H}) ;{ }^{13} \mathrm{C}$ NMR $\delta 170.1,143.5,131.2,125.4,123.3,120.0,117.0,26.2,26.1,24.6,17.5$; MS (EI): $m / z\left[\mathrm{M}^{+}\right]$calcd. for $\mathrm{C}_{13} \mathrm{H}_{18} \mathrm{~N}_{2} \mathrm{O}: 218.14$, found: 218.1; Anal. Calc. for $\mathrm{C}_{13} \mathrm{H}_{18} \mathrm{~N}_{2} \mathrm{O}: \mathrm{C}$, 71.53; H, 8.31; N, 12.83. Found: C, 71.19; H, 8.31; N, 13.20.

(2-Amino-6-methylphenyl)(pyrrolidin-1-yl)methanone (5c). White solid (0.192 g, 94\%); mp 78-79 ${ }^{\circ} \mathrm{C}$; IR $v_{\max }(\mathrm{KBr}) 3430.8,3330.5,3229.2,1617.0 \mathrm{~cm}^{-1} ;{ }^{1} \mathrm{H} \mathrm{NMR}\left(\mathrm{CDCl}_{3}\right) \delta 7.05(\mathrm{t}, J 7.8$ $\mathrm{Hz}, 1 \mathrm{H}), 6.63(\mathrm{~d}, J 7.5 \mathrm{~Hz}, 1 \mathrm{H}), 6.56(\mathrm{~d}, J 8.0 \mathrm{~Hz}, 1 \mathrm{H}), 3.79$ (br s, 2H), $3.73-3.64$ (m, 2H), 3.28 $-3.23(\mathrm{~m}, 1 \mathrm{H}), 3.19-3.14(\mathrm{~m}, 1 \mathrm{H}), 2.24(\mathrm{~s}, 3 \mathrm{H}), 2.02-1.88(\mathrm{~m}, 4 \mathrm{H}) ;{ }^{13} \mathrm{C} \mathrm{NMR} \delta 170.0,143.4$, 135.1, 130.0, 125.4, 121.0, 114.2, 47.6, 45.5, 26.1, 24.8, 19.1; MS (EI): $\mathrm{m} / \mathrm{z}\left[\mathrm{M}^{+}\right]$calcd. for $\mathrm{C}_{12} \mathrm{H}_{16} \mathrm{~N}_{2} \mathrm{O}$ : 204.12, found: 204.0; Anal. Calc. for $\mathrm{C}_{12} \mathrm{H}_{16} \mathrm{~N}_{2} \mathrm{O}: \mathrm{C}, 70.56 ; \mathrm{H}, 7.90 ; \mathrm{N}, 13.71$. Found: C, 70.92; H, 8.17; N, 13.67 .

(2-Amino-5-chlorophenyl) (morpholino) methanone (5d). Pale yellow solid (0.216 g, 90\%); mp 130-131 ${ }^{\circ} \mathrm{C}$; IR $v_{\max }(\mathrm{KBr}) 3424.0,3337.2,3236.9,1617.0 \mathrm{~cm}^{-1} ;{ }^{1} \mathrm{H} \mathrm{NMR}\left(\mathrm{CDCl}_{3}\right) \delta 7.14$ (dd, J 8.6, $2.3 \mathrm{~Hz}, 1 \mathrm{H}), 7.05$ (d, J $2.3 \mathrm{~Hz}, 1 \mathrm{H}), 6.67$ (d, J 8.6 Hz, 1H), 4.35 (br s, 2H), $3.72-3.64$ $(\mathrm{m}, 8 \mathrm{H}) ;{ }^{13} \mathrm{C}$ NMR $\delta 169.6,145.2,131.5,128.2,122.9,121.0,118.7,67.4(2 \mathrm{C}) ; \mathrm{MS}(\mathrm{EI}): \mathrm{m} / z$ $\left[\mathrm{M}^{+}\right]$calcd. for $\mathrm{C}_{11} \mathrm{H}_{13} \mathrm{ClN}_{2} \mathrm{O}_{2}$ : 240.06, found: 240.0; Anal. Calc. for $\mathrm{C}_{11} \mathrm{H}_{13} \mathrm{ClN}_{2} \mathrm{O}_{2}$ : C, 54.88; $\mathrm{H}$, 5.83; N, 11.64. Found: C, 54.89; H, 6.01; N, 11.34.

(2-Amino-5-iodophenyl)(pyrrolidin-1-yl)methanone (5e). Brown solid (0.280 g, 89\%); mp 134-135 ${ }^{\circ} \mathrm{C}$; IR $v_{\max }(\mathrm{KBr}) 3417.2,3322.7,3208.0,1645.9 \mathrm{~cm}^{-1} ;{ }^{1} \mathrm{H}$ NMR $\left(\mathrm{CDCl}_{3}\right) \delta 7.49(\mathrm{~d}, J$ $2.5 \mathrm{~Hz}, 1 \mathrm{H}), 7.42$ (dd, $J$ 8.5, $2.0 \mathrm{~Hz}, 1 \mathrm{H}), 6.52$ (d, J $8.5 \mathrm{~Hz}, 1 \mathrm{H}), 4.58$ (br s, 2H), 3.63 (s, 2H), 3.49 (s, 2H), 1.97 (s, 2H), 1.92 (s, 2H); ${ }^{13} \mathrm{C}$ NMR $\delta$ 167.8, 145.6, 139.1, 136.2, 122.9, 118.8, 49.6, 46.1, 26.4, 24.4; $\mathrm{MS}(\mathrm{EI}): \mathrm{m} / z\left[\mathrm{M}^{+}\right]$calcd. for $\mathrm{C}_{11} \mathrm{H}_{13} \mathrm{IN}_{2} \mathrm{O}: 316.00$, found: 315.9; Anal. Calc. for $\mathrm{C}_{11} \mathrm{H}_{13} \mathrm{IN}_{2} \mathrm{O}$ : C, 41.79; H, 4.15; N, 8.86. Found: C, 42.07; H, 4.61; N, 8.60.

2-Amino-3,5-dibromo- $\boldsymbol{N}, \boldsymbol{N}$-diethylbenzamide (5f). Colourless oil (0.312 g, 89\%); IR $v_{\max }$ (KBr) 3452.9, 3344.9, $1617.0 \mathrm{~cm}^{-1} ;{ }^{1} \mathrm{H} \mathrm{NMR}\left(\mathrm{CDCl}_{3}\right) \delta 7.53(\mathrm{~d}, J 2.2 \mathrm{~Hz}, 1 \mathrm{H}), 7.13(\mathrm{~d}, J 2.2 \mathrm{~Hz}$, 1H), 4.62 (s, 2H), 3.41 (br s, 4H), 1.17 (br s, 6H); ${ }^{13} \mathrm{C}$ NMR $\delta 168.2,141.7,135.1,128.6,123.6$, 111.0, 108.5, 43.4, 39.8, 39.5, 13.5; MS (EI): $m / z\left[\mathrm{M}^{+}\right]$calcd. for $\mathrm{C}_{11} \mathrm{H}_{14} \mathrm{Br}_{2} \mathrm{~N}_{2} \mathrm{O}$ : 347.94, found: 348.0; Anal. Calc. for $\mathrm{C}_{11} \mathrm{H}_{14} \mathrm{Br}_{2} \mathrm{~N}_{2} \mathrm{O}$ : C, 37.74; H, 4.03; N, 8.00. Found: C, 37.69; H, 4.44; N, 7.85 .

(2-Amino-3,5-diiodophenyl) (morpholino) methanone (5g). White solid (0.440 g, 96\%); mp 128-130 ${ }^{\circ} \mathrm{C}$; IR $v_{\max }(\mathrm{KBr}) 3467.4,3378.7,3336.2,1631.5 \mathrm{~cm}^{-1} ;{ }^{1} \mathrm{H}$ NMR $\left(\mathrm{CDCl}_{3}\right) \delta 7.95(\mathrm{~d}, J$ $1.0 \mathrm{~Hz}, 1 \mathrm{H}), 7.32(\mathrm{~d}, J 1.5 \mathrm{~Hz}, 1 \mathrm{H}), 4.84(\mathrm{br} \mathrm{s}, 2 \mathrm{H}), 3.71-3.63(\mathrm{~m}, 8 \mathrm{H}) ;{ }^{13} \mathrm{C}$ NMR $\delta 167.5$, 147.3, 145.3, 136.0, 120.8, 87.1, 77.7, 66.9, 31.0; $\mathrm{MS}(\mathrm{EI}): \mathrm{m} / z\left[\mathrm{M}^{+}\right]$calcd. for $\mathrm{C}_{11} \mathrm{H}_{12} \mathrm{I}_{2} \mathrm{~N}_{2} \mathrm{O}_{2}$ : 457.89, found: 457.9; Anal. Calc. for $\mathrm{C}_{11} \mathrm{H}_{12} \mathrm{I}_{2} \mathrm{~N}_{2} \mathrm{O}_{2}$ : C, 28.84; H, 2.64; N, 6.12. Found: C, 29.03; $\mathrm{H}, 2.86 ; \mathrm{N}, 6.45$.

2-Amino- $N, N$-diethyl-3,4,5-trimethoxybenzamide (5h). Dark yellow oil (0.262 g, 93\%); IR $v_{\max }(\mathrm{KBr}) 3452.9,3351.7,1631.5 \mathrm{~cm}^{-1} ;{ }^{1} \mathrm{H} \mathrm{NMR}\left(\mathrm{CDCl}_{3}\right) \delta 6.48(\mathrm{~s}, 1 \mathrm{H}), 3.91(\mathrm{~s}, 3 \mathrm{H}), 3.90(\mathrm{~s}$, $3 \mathrm{H}), 3.80(\mathrm{~s}, 3 \mathrm{H}), 3.45$ (br s, 6H), $1.23(\mathrm{~s}, 6 \mathrm{H}) ;{ }^{13} \mathrm{C}$ NMR $\delta 170.2,145.2,143.7,141.9,133.1$, 116.5, 106.3, 60.9, 60.5, 56.7, 13.6; MS (EI): $m / z\left[\mathrm{M}^{+}\right]$calcd. for $\mathrm{C}_{14} \mathrm{H}_{22} \mathrm{~N}_{2} \mathrm{O}_{4}$ : 282.15, found: 
282.1; Anal. Calc. for $\mathrm{C}_{14} \mathrm{H}_{22} \mathrm{~N}_{2} \mathrm{O}_{4}$ : C, 59.56; H, 7.85; N, 9.92. Found: C, 59.19; H, 7.51; N, 10.29 .

\section{Acknowledgements}

This work is dedicated to Professor Cemil Öğretir, who died on $19^{\text {th }}$ January, 2011. It is gratefully acknowledged that this work was supported financially by Anadolu University (Project No: 1002F88) and we are also grateful to AUBIBAM for providing ${ }^{1} \mathrm{H}$ and ${ }^{13} \mathrm{C}$ NMR spectra.

\section{References}

1. Bettelheim, F. A.; March, J. Introduction to General, Organic and Biochemistry, 5th Ed.; Sounders College Publishing: Fort Worth, 1998; pp 492-508.

2. Chung, Y. J.; Jung, Y. S.; Seong, C. M.; Park, N. S. Bull. Korean Chem. Soc. 1998, 19 (10), 1117.

3. (a) Siddiqui, I. R.; Siddique, S. A.; Srivastava, V.; Singh, P. K.; Singh, J. Arkivoc 2008, (xii), 277. (b) Subba Reddy, B. V.; Venkateswarlu, A.; Madan, C.; Vinu, A. Tetrahedron Lett. 2011, 52, 1891. (c) Loseva, M. V.; Bolotin, B. M. Chemistry of Heterocyclic Compounds 1972, 10, 1212.

4. Shariat, M.; Abdollahi, S. Molecules 2004, 9, 705.

5. Nishimura, T.; Iino, T.; Mitsuya, M.; Bamba, M.; Watanabe, H.; Tsukahara, D.; Kamata, K.; Sasaki, K.; Ohyama, S.; Hosaka, H.; Futamura, M.; Nagata, Y.; Eiki, J. Bioorg. Med. Chem. Lett. 2009, 19, 1357.

6. Shaabani, A.; Maleki, A.; Mofakham, H.; Khavasi, H. R. J. Comb. Chem. 2008, 10, 883.

7. (a) Tani, J.; Yamada, Y.; Oine, T.; Ochiai, T.; Ishida, R.; Inoue, I. J. Med. Chem. 1979, 22, 95. (b) Kim, D.; Wang., L.; Hale, J. J.; Lynch, C. L.; Budhu, R. J.; MacCoss, M.; Mills, S. G.; Malkowitz, L.; Gould, S. L.; DeMartino, J. A.; Springer, M. S.; Hazuda, D.; Miller, M.; Kessler, J.; Hrin, R. C.; Carver, G.; Carella, A.; Henry, K.; Lineberger, J.; Schleif, W. A.; Emini, E. A. Bioorg. Med. Chem. Lett. 2005, 15, 2129. (c) Wright, W. B.; Tomcufcik, A. S.; Chan, P. S.; Marsico, J. W.; Press, J. B. J. Med. Chem. 1987, 30, 2277. (d) Lee, J. S.; Oh, Y. S.; Lim, J. K.; Yang, W. Y.; Kim, I. H.; Lee, C. W.; Chung, Y. H.; Yoon, S. J. Synth. Commun. 1999, 29, 2547. (e) Larsen, U. S.; Begtrup, M.; Martiny, L. J. Label. Compd. Radiopharm. 2005, 48, 429. (f) Asano, T.; Yoshikawa, T.; Usui, T.; Yamamoto, H.; Yamamoto, Y.; Uehara, Y.; Nakamura, H. Bioorg. Med. Chem. 2004, 12, 3529.

8. Rivière-Baudet, M.; Morère, A.; Dias, M. Tetrahedron Lett. 1992, 33, 6453.

9. Sellstedt, J. H.; Guinosso, C. J.; Begany, A. J.; Bell, S. C.; Rosenthale, M. J. Med. Chem. 1975, 18, 926. 
10. Asano, T.; Yoshikawa, T.; Nakamura, H.; Ueharac, Y.; Yamamoto, Y. Bioorg. Med. Chem. Lett. 2004, 14, 2299.

11. Staiger, R. P.; Miller, E. B. J. Org. Chem. 1959, 24, 1214.

12. Allison, B. D.; Phuong, V. K.; McAtee, L. C.; Rosen, M.; Morton, M.; Prendergast, C.; Barrett, T.; Lagaud, G.; Freedman, J.; Li, L.; Wu, X.; Venkatesan, H.; Pippel, M.; Woods, C.; Rizzolio, M. C.; Hack, M.; Hoey, K.; Deng, X.; King, C.; Shankley, N. P.; Rabinowitz, M. H. J. Med. Chem. 2006, 49, 6371.

13. (a) Katritzky, A. R.; Mohapatra, P. P.; Huang, L. Arkivoc 2008, (ix), 62. (b) Katritzky, A. R.; Cai, C.; Suzuki, K.; Singh, S. K. J. Org. Chem. 2004, 69, 811.

14. (a) Katritzky, A. R.; He, H-Y.; Suzuki, K. J. Org. Chem. 2000, 65, 8210. (b) Katritzky, A. R.; Suzuki, K.; Wang, Z. Synlett 2005, 11, 1656. (c) Celik, İ.; Abdel-Fattah, A. A. A. Tetrahedron 2009, 65, 4923.

15. (a) Katritzky, A. R.; Suzuki, K.; Singh, S. K. CCACAA 2004, 77 (1-2), 175. (b) Wang, X.; Zhang, Y. Tetrahedron Lett. 2002, 43, 5431.

16. (a) Katritzky, A. R.; Shestopalov, A. A.; Suzuki, K. Synthesis 2004, 11, 1806. (b) Lin, S-M.; Zhang, J-L.; Chen, J-K.; Gao, W-X.; Ding, J-C.; Su, W-K.; Wu, H-Y. J. Braz. Chem. Soc. 2010, 21, 1616.

17. Katritzky, A. R.; Singh, S. K.; Cai, C.; Bobrov, S. J. Org. Chem. 2006, 71 (9), 3364.

18. Katritzky, A. R.; Meher, N. K.; Cai, C.; Singh, S. K. Rev. Soc. Quím. Méx. 2004, 48, 275.

19. Katritzky, A. R.; Zhang, Y.; Singh, S. K. Synthesis 2003, 18, 2795.

20. Katritzky, A. R.; Vakulenko, A. V.; Jain, R. Arkıvoc 2003, (xiv), 131.

21. (a) Zhao, H.; Fu, H.; Qiao, R. J. Org. Chem. 2010, 75, 3311. (b) Cheng, X.; Vellalath, S.; Goddard, R.; List, B. J. Am. Chem. Soc. 2008, 130, 15786.

22. Staiger, R. P.; Wagner, E. C. J. Org. Chem. 1948, 13, 347.

23. Javier, B. O.; Manuel, A. L. PCT Int. Appl., 2001066 519, 2001.

24. Varasi, M.; Thaler, F.; Abate, A.; Carenzi, G.; Minucci, S. PCT Int. Appl., 2009127 609, 2009.

25. Sardon, T.; Cottin, T.; Xu, J.; Giannis, A.; Vernos, I. ChemBioChem 2009, 10, 464.

26. Wong, N. C. W.; Tucker, J. E. L. ; Hansen, H. C.; Chiacchia, F. S.; McCaffrey, D. U.S. Pat. Appl. Publ., 20080188 467, 2008.

27. Eger, K. Arch. Pharm. 1981, 314, 176.

28. Petrov, J. S.; Andreev, G. N. Org. Prep. Proced. Int. 2005, 37, 560.

29. Kornet, M. J. J. Heterocycl. Chem. 1992, 29, 103.

30. Barlaam, B. C.; Foote, K. M.; Ple, P. PCT Int. Appl., 2009153589, 2009.

31. Ogita, H.; Isobe, Y.; Takaku, H.; Sekine, R.; Goto, Y.; Misawa, S.; Hayashi, H. Bioorg. Med. Chem. Lett. 2001, 11, 549. 